Research article urn:lsid:zoobank.org:pub:D7F9AFC7-B607-4D4F-B23E-F844C1776EED

\title{
Taxonomic study of the genus Microplitis Förster, 1862 (Hymenoptera, Braconidae, Microgastrinae) from Iran
}

\author{
Parisa ABDOLI ${ }^{1}$, Ali Asghar TALEBI ${ }^{2, *}$, \\ Jose FERNANDEZ-TRIANA ${ }^{3}$ \& Samira FARAHANI ${ }^{4}$ \\ ${ }^{1,2}$ Department of Entomology, Faculty of Agriculture, Tarbiat Modares University, Tehran, Iran. \\ ${ }^{3}$ Canadian National Collection of Insects, Ottawa, Canada. \\ ${ }^{4}$ Research Institute of Forests and Rangelands, \\ Agricultural Research Education and Extension Organization (AREEO), Tehran, Iran. \\ ${ }^{*}$ Corresponding author: talebia@modares.ac.ir \\ ${ }^{1}$ Email: P.abdoli@modares.ac.ir \\ ${ }^{3}$ Email: jose.fernandez@canada.ca \\ ${ }^{4}$ Email: s.farahani@rifr-ac.ir \\ 2 ๑ https://orcid.org/0000-0001-5749-6391 \\ $3 \odot$ https://orcid.org/0000-0003-0425-0309 \\ ${ }^{4} \odot$ https://orcid.org/0000-0002-6897-0631

\footnotetext{
${ }^{1}$ urn:1sid:zoobank.org:author:57404069-C271-4439-AE0B-2EAFC0D03BB7

${ }^{2}$ urn:1sid:zoobank.org:author:71CB13A9-F9BD-4DDE-8CB1-A495036975FE

${ }^{3}$ urn:lsid:zoobank.org:author:6D9B3500-93B8-4DC8-A732-A6FB2B5A930B
} \\ ${ }^{4}$ urn:lsid:zoobank.org:author:423DEB84-81C3-4179-BDE2-88A827CD4865
}

\begin{abstract}
The genus Microplitis Förster, 1862 (Hymenoptera, Braconidae, Microgastrinae) was studied from northern Iran. Specimens were collected using Malaise traps during 2010-2011. A total of 13 species were collected and identified, of which six species are recorded for the first time from Iran: M. cebes Nixon, 1970, M. docilis Nixon, 1970, M. eremitus Reinhard, 1880, M. kaszabi Papp, 1980, M. pallidipennis Tobias, 1964 and M. varipes (Ruthe, 1860). Two species M. kaszabi and M. pallidipennis are new records for the west Palaearctic region. A new species, Microplitis alborziensis Abdoli \& Talebi sp. nov., is described and illustrated. The number of species of Microplitis in Iran is now raised from 17 to 24 . A faunistic list, an identification key to all known Iranian species and brief diagnoses and illustrations for all species that have been collected in this study are provided. The validity of the new species is supported by DNA barcoding.
\end{abstract}

Keywords. Taxonomy, new records, new taxa, identification key, Microgastrinae, Palaearctic.

Abdoli P., Talebi A.A., Fernandez-Triana J. \& Farahani S. 2021. Taxonomic study of the genus Microplitis Forster, 1862 (Hymenoptera, Braconidae, Microgastrinae) from Iran. European Journal of Taxonomy 744: 83-118. https://doi.org/10.5852/ejt.2021.744.1305 


\section{Introduction}

Microgastrinae Förster, 1862 is a large and diverse subfamily in the Braconidae with about 3000 described species worldwide (Fernandez-Triana et al. 2020). This subfamily is one of the most important groups of parasitoids in terms of both species richness and economic importance (Rodriguez et al. 2013). Until 1862, all described species in Microgastrinae were assigned to the genus Microgaster Latreille, 1804. Förster (1862) erected two additional genera for Microgastrinae: Microplitis and Apanteles (Whitfield et al. 2002).

The genus Microplitis Förster, 1862 comprises 192 described species worldwide including 108 species in the Palaearctic region (Fernandez-Triana et al. 2020). Species of Microplitis are beneficial parasitoid wasps, predominantly attacking exposed Macrolepidoptera and many of their hosts live fully exposed on vegetation throughout their larval stages. Larvae of species of Microplitis are essentially hemolymph and fat-body feeders, and in many cases their hosts remain alive for several days after the parasitoid larvae exit the host body, which usually takes place from the central or posterior abdominal segments of the caterpillar (Shaw \& Huddleston 1991).

The genus Microplitis has been revised and keyed in the Palaearctic by several authors (Fahringer 1937; Nixon 1968, 1970; Papp 1986, 1987, 1990; Tobias 1986; Xu \& He 2003; Chen \& Song 2004; Kotenko 2007; Zhang et al. 2019), and also in other regions of the world such as the Australian (Austin \& Dangerfield 1993), Afrotropical (De Saeger 1944), Oriental (Ranjith et al. 2015) and Costa Rican (Fernandez-Triana et al. 2015).

The fauna of some subfamilies of Iranian Braconidae was already catalogued (Rakhshani et al. 2007; Farahani et al. 2012, 2014a, 2014b, 2014d, 2015, 2016; Ameri et al. 2014, 2015; Ghahari \& Beyarslan 2017) but Microgastrinae has been poorly studied (Farahani et al. 2014c; Karimi-Malati et al. 2014; Gadallah et al. 2015; Ghafouri Moghaddam et al. 2018; Samin et al. 2018; Abdoli et al. 2019a, 2019b; Zargar et al. 2019a, 2019b, 2019c). Prior to this study, only 17 species of Microplitis had been recorded from Iran (Farahani et al. 2014c; Karimi-Malati et al. 2014; Gadallah et al. 2015; Samin et al. 2018, 2019; Belokobylskij et al. 2019; Fernandez-Triana et al. 2020). The objective of this study is to improve our knowledge about the genus Microplitis from Iran.

\section{Material and methods}

Specimens for the present study were collected using Malaise traps from March to November of 2010 and 2011 in northern Iran (i.e., Alborz, Guilan, Mazandaran, Qazvin and Tehran Provinces) (Fig. 1). Malaise traps were placed in a range of different habitats such as forests, rangelands and orchards. The specimens were identified using the keys of Telenga (1955), Papp (1984), Tobias (1986) and Kotenko (2007). Morphological terminology follows Karlsson \& Ronquist (2012) for various body parts and Wharton et al. (1997) for wing venation. The specimens were photographed with a Keyence VHX1000 Digital microscope, using a lens with a range of 13-130×. Multiple images through the focal plane were taken of a structure, and latter, these were combined to produce a single in-focus image. The software associated with the Keyence system produced the focused images taken with that camera. The measurements were done using an Olympus ${ }^{\mathrm{TM}}$ SZX9 stereo microscope equipped with a graticule. The specimens are deposited at the TMUC.

DNA barcoding of new species focused on the sequencing of a short, standardized portion of the mitochondrial cytochrome c oxidase I gene (COI). The sample in this study (a paratype) had some legs removed for DNA. DNA extraction method follows Brewster \& Paoli (2013). The COI gene was amplified using primers (LCO1490-HC02198) following standard protocols (Folmer et al. 1994). The barcode data is stored in the National Center for Biotechnology Information. A map with the Iranian 
provinces where the species of Microplitis were collected in this study was generated using Simple Mapper (Shorthouse 2010).

\section{Abbreviations}

F2, F8, F15 = flagellomeres 2, 8 and 15

$\mathrm{OD}=$ ocellus diameter (maximum diameter of anterior ocellus)

OOL $=$ ocello-ocular line (minimum distance between posterior ocelli and eyes)

$\mathrm{POL}=$ posterior ocellar line (minimum distance between posterior ocelli)

$\mathrm{T} 1, \mathrm{~T} 2, \mathrm{~T} 3=$ tergites 1,2 and 3

$\mathrm{T} 2+=\mathrm{T} 2$ to end of terga

\section{Repositories}

$\mathrm{CNC}=$ Canadian National Collection of Insects, Agriculture and Agri-food Canada

TMUC $=$ Insect Collection of the Department of Entomology, Tarbiat Modares University, Tehran, Iran

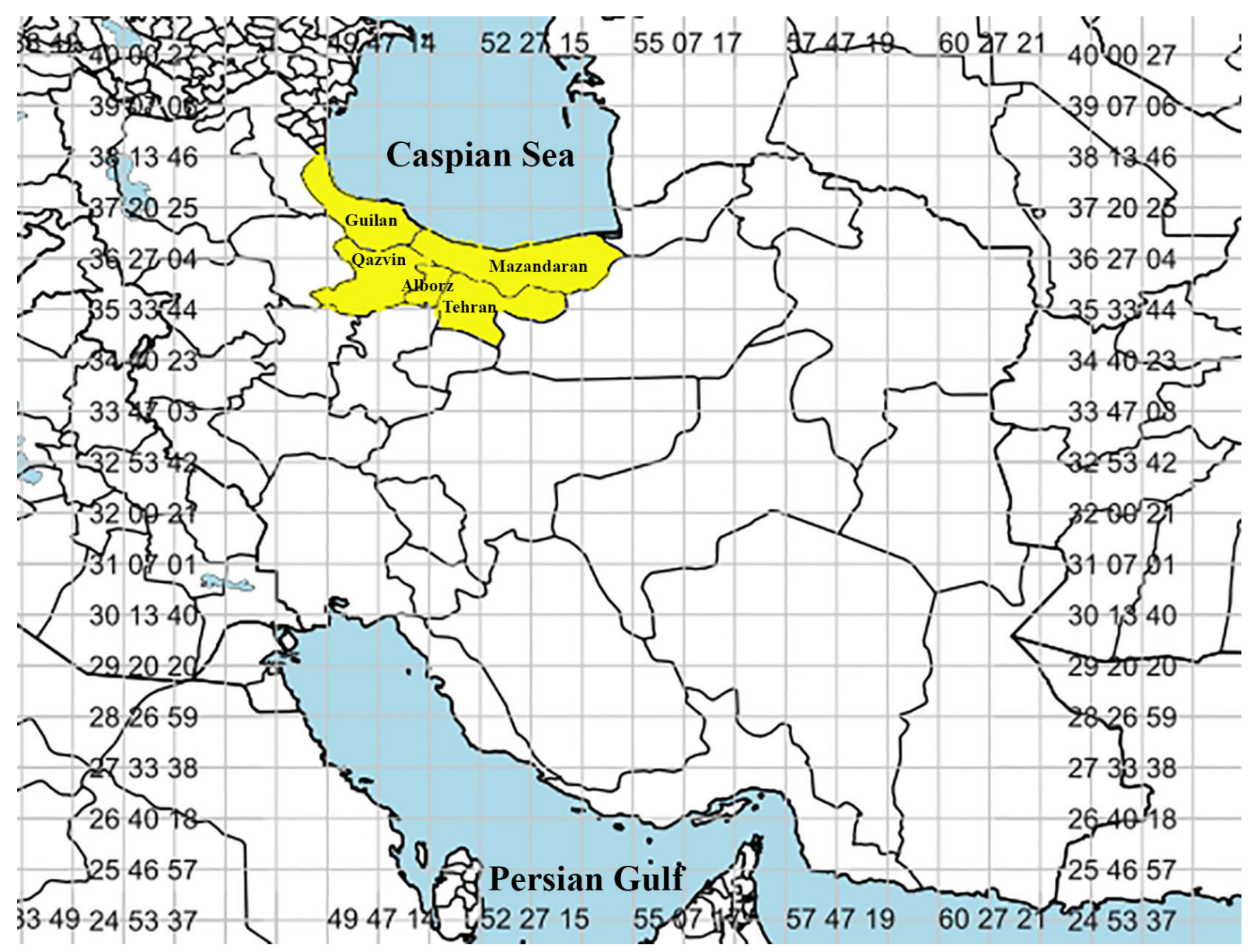

Fig. 1. Map of Iran: Alborz, Qazvin, Tehran, Guilan and Mazandaran Provinces, where specimens have been collected in this study. 


\title{
Results
}

\author{
Class Insecta Linnaeus, 1758 \\ Order Hymenoptera Linnaeus, 1758 \\ Superfamily Ichneumonoidea Latreille, 1802 \\ Family Braconidae Nees, 1811 \\ Subfamily Microgastrinae Förster, 1862 \\ Tribe Microplitini Mason, 1981
}

Genus Microplitis Förster, 1862

Microplitis Förster, 1862: 245 (type species: Microgaster sordipes Nees, 1834: 167 (by original designation, holotype + , Klugiano Museum)).

\section{Diagnosis}

Members of this genus are distinguished by having anteromesoscutum densely sculptured, seldom shining, often with notauli which sometimes are strongly defined; propodeum evenly curved in profile, completely rugose and often with a median longitudinal carina but never with any indication of areola; fore wing always with a closed areolet; vannal lobe of the hind wing convex and setose; metacoxa small, not reaching past the posterior margin of T1; metatibial spurs shorter than one-half of metabasitarsus; mesotibial spurs shorter than mesobasitarsus; $\mathrm{T} 1$ of variable shape, from widening to narrowing posteriorly and usually sculptured; $72+$ smooth, rarely weakly sculptured and often with a weakly delimited trapezoidal median area; T3 always longer than T2, the transverse groove separating them frequently not, or only poorly, defined; hypopygium usually rather small but sometimes elongated medially, rarely conspicuously so, occasionally truncate or medially emarginated; ovipositor and its sheath usually short (Mason 1981).

\section{Identification key to the Iranian species of Microplitis Förster, 1862}

The following identification key is mostly based on Papp (1984), Tobias (1986) and Kotenko (2007) but modified for Iranian species and including one species newly described in this paper.

1. T1 distinctly broadening posteriorly, T1 length almost equal to its posterior width (Figs 8F, 9F); T2 distinctly rugoluse

- T1 parallel- to sub-parallel-sided or weakly broadening posteriorly (Figs 2F, 3F, 4F, 5F, 6F, 7F, 10F, $11 \mathrm{~F}, 12 \mathrm{~F}, 13 \mathrm{~F}, 14 \mathrm{~F}), \mathrm{T} 1 \mathrm{much}$ longer than posterior width; $\mathrm{T} 2$ with variable sculptures 4

2. Body mostly reddish yellow, except for black head (Fig. $8 \mathrm{~A}-\mathrm{F}$ ); vein R1 more than $0.5 \times$ pterostigma length; pterostigma length $2.00 \times$ its width (Fig. 8D) M. ochraceus Szepligeti, 1896

- Body black; other characters variable 3

3. Antenna shorter than body; F15 length $1.50 \times$ its width; hypopygium in lateral view strongly developed, with characteristically pointed apex; tegula black M. xanthopus Ruthe, 1860

- Antenna as long as body; F15 length $2.00 \times$ its width; hypopygium normal; tegula yellow or reddish yellow M. pallidipennis Tobias, 1964

4. T1 length more than $1.70 \times(1.70-3.00)$ its maximum width, $\mathrm{T} 1$ usually parallel-sided or narrowing posteriorly (Figs $2 \mathrm{~F}, 3 \mathrm{~F}, 7 \mathrm{~F}, 10 \mathrm{~F}, 13 \mathrm{~F}$ )

- T1 length $1.60 \times$ or less $(1.40-1.60)$ its maximum width, T1 more or less broadening posteriorly (Figs 4F, 5F, 6F, 11F, 12F, 14F) 


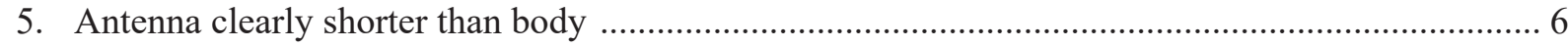

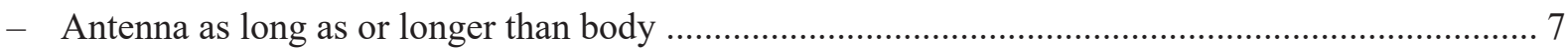

6. In dorsal view, head width (between temples) slightly broader than head width (between eyes); tegula yellow to brown; vein R1 $0.5 \times$ pterostigma length; vein $1 \mathrm{CUb} 2.50 \times$ as long as vein $1 \mathrm{CUa}$; vein $1 \mathrm{CUb}$ and vein $\mathrm{r}-\mathrm{m}$ slightly curved; vein $\mathrm{r}$ shorter than vein $2 \mathrm{RS}$; $\mathrm{T} 1$ weakly broadening posteriorly

M. aduncus (Ruthe, 1860)

- In dorsal view, head width (between temples) similar to head width (between eyes) (Fig. 2B); tegula black; vein R1 more than $0.5 \times$ pterostigma length; vein $1 \mathrm{CUb} 2.00 \times$ as long as vein $1 \mathrm{CUa}$; vein $1 \mathrm{CUb}$ and vein $\mathrm{r}-\mathrm{m}$ straight; vein $\mathrm{r}$ slightly longer than vein 2RS (Fig. 2D); T1 parallel-sided or weakly barrel-shaped (Fig. 2F)

M. alborziensis Abdoli \& Talebi sp. nov.

7. Tegula and metafemur dark brown to black 8

- Tegula and metafemur yellow to reddish yellow 9

8. Pterostigma with large anterior pale spot; T1 medially smooth to uneven, shiny, laterally rugorugulose M. sofron Nixon, 1970

- Pterostigma without or with a small anterior yellow spot; T1 rugose to coriaceous, matte

M. cebes Nixon, 1970

9. Metasoma yellow or reddish yellow, at least last 2-3 segments more or less darkening M. rufiventris Kokujev, 1914

- Metasoma black or dark brown, at least first 2-3 segments lightening

10. T1 length more than $2.20 \times(2.50-3.50)$ its maximum width; antenna dark, brown to black; tergites black, sometimes T2 and T3 yellow to brown; metacoxa yellow to reddish yellow; in fore wing, vein $1 \mathrm{CUb} 2.00 \times$ as long as vein $1 \mathrm{CUa}$ (Fig. 13D); in hind wing, vein $2 \mathrm{r}-\mathrm{m}$ about $1.75-1.90 \times$ as long as vein $1 \mathrm{r}-\mathrm{m}$ (Fig. 13E)

M. tuberculifer (Wesmael, 1837)

- T1 length $2.00 \times$ (or less) its maximum width, other characters variable

11. $6^{\text {th }}$ sternite distinctly developed, produced beyond apex of metasoma; $\mathrm{T} 1$ reddish yellow (sometimes with blackish suffusion) and narrowed toward apex and base M. fulvicornis (Wesmael, 1837)

- $6^{\text {th }}$ sternite not produced beyond apex of metasoma; T1 black or dark brown and narrowed toward apex or base

12. Antenna usually light coloured (yellow to brownish yellow), distally darkening; tergites and metacoxa black; in fore wing, vein 1CUb $3.00 \times$ as long as vein 1CUa (Fig. 7D); in hind wing, vein $2 \mathrm{r}-\mathrm{m}$ about $1.40-1.60 \times$ as long as vein $1 \mathrm{r}-\mathrm{m}$ (Fig. 7D) M. mandibularis Thomson, 1895

- Antenna always dark brown or black (scape reddish yellow or black); other characters variable .....

13. T1 in posterior third sculptured, matte, in basal half smooth; pterostigma entirely dark brown, without anterior pale spot; basal one third of wing with light-coloured veins, the rest with darkened veins; scape reddish yellow, in contrasts with a dark-coloured flagellum; T2 and T3 black

M. marshallii Kokujev, 1898

- T1 almost entirely sculptured; pterostigma with anterior pale spot; wing all smoky, with darkened veins; scape dark-coloured, does not contrast in colour with the flagellum; T2 and T3 often light coloured

M. mediator Haliday, 1834 
14. Antenna shorter than body; F15 $1.40-1.50 \times$ as long as wide (Fig. 11A); pterostigma length 2.30 $2.40 \times$ its width, with light yellow spot anteriorly (Fig. 11D); all coxae black to infuscate; metafemur reddish brown, brown to black; tegula dark brown to black M. spectabilis (Haliday, 1834)

- Antenna longer than body; other characters variable 15

15. T2 rugulose or sub-rugulose (Fig. 12E); frons polished (Fig. 12D) M. spinolae (Nees, 1834)

- T2 smooth, frons not polished (if T2 uneven to shrivelled then frons sculptured)

16. Tegula and metafemur yellow or reddish yellow 17

- Tegula black and metafemur variable coloured

17. Mesoscutellar disc weakly and dispersedly punctate to smooth, with medio-longitudinal keel anteriorly which is rather short and low; pterostigma without anterior pale spot; T2 rather smooth to polished, though frequently uneven to shrivelled, less frequently rugulose

M. deprimator (Fabricius, 1798)

- Mesoscutellar disc coriaceous to rugulose, without keel anteriorly, pterostigma with or without anterior yellow spot

18. T2 and T3 yellow or reddish yellow (Fig. 6F); pterostigma with anterior yellow spot (Fig. 6D) ......

M. kaszabi Papp, 1980

- T2 and T3 black or brown, sometimes with small yellow areas; pterostigma without anterior pale spot (Fig. 5D) M. eremitus Reinhard, 1880

19. Metafemur yellow; tergites black, sometimes $\mathrm{T} 2$ and $\mathrm{T} 3$ ferruginous to red

M. scrophulariae Szépligeti, 1898

- Metafemur black or dark brown (sometimes with yellowish patterns); tergites black

20. Mesoscutellar disc strongly rugose (Fig. 4C); scape yellow to reddish yellow, contrasting with dark brown flagellum; pterostigma without anterior pale spot (Fig. 4D) M. docilis Nixon, 1970

- Mesoscutellar disc with usual sculpture, finely to densely punctate, matte or shiny; scape black or brown, similar colour to flagellum; pterostigma with or without anterior pale spot

21. Fore wing areolet relatively very large and almost pentagonal; lateral and median lobes of anteromesonotum densely shagreened, dull; pterostigma black, with a small pale spot anteriorly ...

M. decipiens Prell, 1925

- Fore wing areolet relatively smaller and triangular; lateral and median lobes of anteromesonotum rugulose to rugose; pterostigma with or without pale spot anteriorly

22. Pterostigma with distinct pale spot anteriorly (Fig. 14D); F15 length $2.00 \times$ its width; body relatively smaller, its length at most $3.50 \mathrm{~mm}$, usually $2.80-3.50 \mathrm{~mm}$

M. varipes (Ruthe, 1860)

- Pterostigma evenly brown or with diffusely pale spot anteriorly; F15 length $1.50 \times$ its width; body relatively bigger, usually $4.00-4.20 \mathrm{~mm}$

M. viduus (Ruthe, 1860)

Microplitis aduncus (Ruthe, 1860)

Microgaster aduncus Ruthe, 1860: 129.

Microgaster brachycera Thomson, 1895: 2252, pl. 13.

\section{Distribution in Iran}

Iran (no locality cited) (Papp 1984). 
Microplitis albipennis Abdinbekova, 1969: 74.

\section{Distribution in Iran}

Khuzestan Province (Samin et al. 2018).

Microplitis alborziensis Abdoli \& Talebi sp. nov. urn:1sid:zoobank.org:act:1D299C75-E2F5-41C5-B275-90253151CEC6

Fig. 2

\section{Diagnosis}

Antenna shorter than body; head in dorsal view more transverse (i.e., its width/length ratio 1.90) and rounded behind eyes; scutoscutellar sulcus distinctly widened, with eight distinct costulae; length of vein $\mathrm{R} 1$ shorter than length of pterostigma; vein $1 \mathrm{CUb} 2.0 \times$ as long as vein $1 \mathrm{CUa}$; vein $\mathrm{r}$ as long as to slightly longer than vein 2RS; T1 length/width ratio 2.0, parallel-sided (or weakly barrel-shaped), rugose to rugulose; tegula, scape and metasomal tergites black; metafemur yellow.

\section{Etymology}

The new species is named after the type locality. The species name 'alborziensis' is an adjective derived from the Alborz Province in Iran.

\section{Material examined}

\section{Holotype}

IRAN - Alborz Province • 9 ; Chalous Road, Sarziarat; 3555'10.38" N, 5106'51.24" E; 1980 m a.s.1.; 22 Jul. 2010; M. Khayrandish leg.; Malaise trap; TMUC-HBMMP0001.

\section{Paratypes}

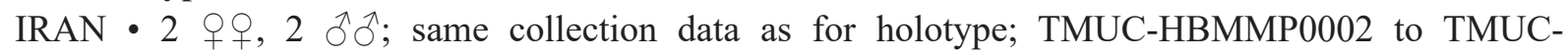
HBMMP0005.

\section{Description}

Female (holotype)

MEASUREMENTS. Body length $3.7 \mathrm{~mm}$; antenna length $3.2 \mathrm{~mm}$; fore wing length $3.4 \mathrm{~mm}$.

HEAD (Fig. 2B). Antenna shorter than body, its segments normally setose; F2, F8, F15 length/width ratio 2.5, 2.0 and 1.5, respectively; head rounded behind eyes; mouthparts not elongated; gena, vertex, upper and lower face (i.e., frons and face, respectively) punctate; clypeus and labrum softly punctate and shiny; basal width of mandible $2.00 \times$ malar length; length of OOL, DO, POL: 0.2, 0.1 and $0.2 \mathrm{~mm}$, respectively.

Mesosoma (Fig. 2C). Anteromesoscutum punctate; notauli weakly defined; scutoscutellar sulcus widened, with eight distinct costulae; mesoscutellar disc coarsely punctate; side of mesoscutellar disc with widened crenulated depression; metanotum coarsely wrinkled; propodeum rugose with a complete median longitudinal carina; pronotum and propleuron coarsely punctate; epicnemial carina absent; anterolateral, dorsolateral and ventrolateral parts of mesopleuron punctate, medially smooth; metapleuron punctate. 
Wings (Fig. 2D-E). Pterostigma length/width ratio 2.4, pterostigma $1.4 \times$ as long as vein R1; vein $\mathrm{R} 1$ as long as or slightly shorter than distance of vein $\mathrm{R} 1$ to vein $3 \mathrm{RSb}$; vein $\mathrm{r}$ slightly longer than vein $2 \mathrm{RS}$; vein $1 \mathrm{M} / \mathrm{m}$-cu ratio 1.9 ; areolet closed; vein $1 \mathrm{CUb} 2.0 \times$ as long as vein $1 \mathrm{CUa}$. Hind wing: vein $\mathrm{M}+\mathrm{CU} / 1-\mathrm{M}$ ratio 0.9 ; vein cu-a slightly curved; vannal lobe fringed anteriorly, with slight setae posteriorly and posterior margin somewhat straight

LEGS (Fig. 2D). Metacoxa finely punctate; length of metafemur, metatibia, metabasitarsus and combined of other metatibial tarsus: $0.9,1.1,0.5$ and $0.7 \mathrm{~mm}$, respectively; metafemur length/width ratio 4.0; metatibial spurs equal or outer spur slightly shorter than inner spur; metatibial inner spur as long as half of metabasitarsus.

Metasoma (Fig. 2F). T1 length $2.0 \times$ its maximum width, parallel-sided or weakly barrel-shaped, rugose to rugulose; ovipositor sheath short; hypopygium sclerotized; abdomen almost as long as combined length of head and thorax.

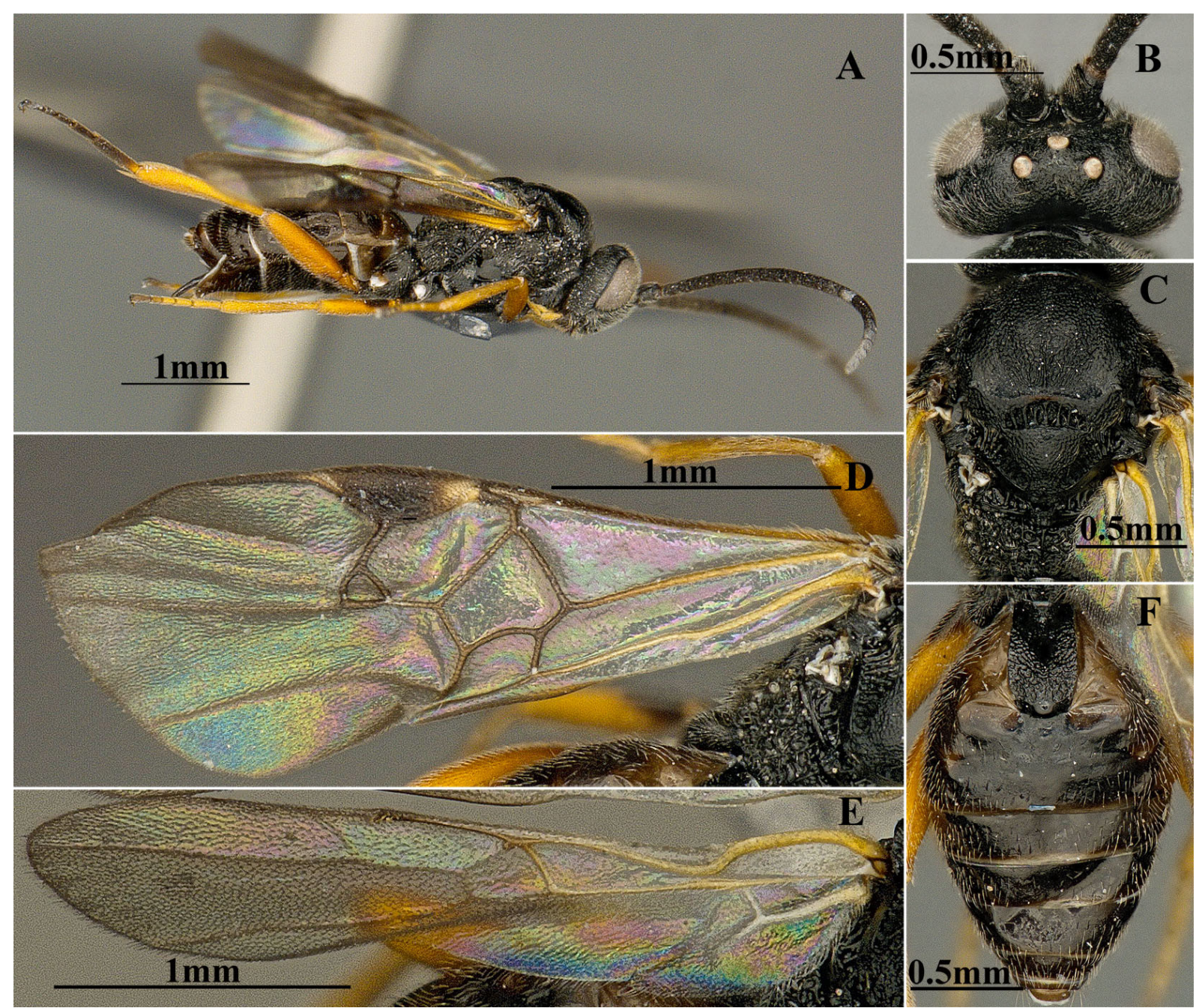

Fig. 2. Microplitis alborziensis Abdoli \& Talebi sp. nov., holotype,, , TMUC-HBMMP0001. A. Habitus, lateral view. B. Head, dorsal view. C. Mesosoma, dorsal view. D. Fore wing. E. Hind wing. F. Metasoma, dorsal view. 
CoLour. Body black; palpi and legs yellow or reddish yellow (except for black coxae and metatarsus); wings with brownish yellow setae; wing venation brown, pterostigma dark brown, with a small basal yellow spot (Fig. 2D).

\section{DNA barcode}

The DNA barcode sequences of Microplitis alborziensis sp. nov. specimen is available at the NCBI database (National Center for Biotechnology Information: https://www.ncbi.nlm.nih.gov/; accession number: MN820452.1).

The available data in NCBI shows that two specimens, Microplitis jft111 (European, Sweden, with accession numbers of NCBI: HM396982.1) and Microplitis jft112 (North American, Alaska, with accession numbers of NCBI: HM397413.1), for which there are DNA barcodes available (all based from specimens in the CNC) differ by $1.7 \%$ base pairs (11 base pairs of difference) and $1.6 \%$ base pairs (10 base pairs of difference), respectively, suggesting that they are different species (see Smith et al. 2013for further details).

\section{Remarks}

Microplitis alborziensis sp. nov. runs to M. tuberculifer (Wesmael, 1937) in the key provided by Telenga (1955) and runs to M. trochanterata (Thomson, 1895) in the key of Tobias (1986) (Tobias' species was later synonymized with $M$. tuberculifer (Broad et al. 2016)). It can, however, be separated from M. tuberculifer by the following characters: (1) T1 length/width ratio is 2.0 vs 2.5-3.0 in M. tuberculifer, (2) antenna shorter than body vs slightly longer than body in M. tuberculifer, (3) tegula black vs reddish yellow to brown in M. tuberculifer.

The new species runs to M. mediator (Haliday, 1834) in the key of Kotenko (2007) from which it can be separated by: (1) head in dorsal view more transverse (i.e., its width/length ratio 1.90) vs head in dorsal view less transverse (i.e., its width/length ratio 1.60-1.70) in M. mediator, (2) hind legs yellow, metasomal tergites and scape black vs hind legs dark brown, metasomal tergites and scape reddish yellow to red in M. mediator.

This species runs to M. aduncus (Ruthe, 1860) in Papp (1984), however, M. aduncus differs from M. alborziensis sp. nov. as follows: in M. alborziensis sp. nov. meso- and metafemora are yellow, vein cu-a in hind wing is slightly curved, and T1 is rugose to rugulose, whereas in M. aduncus meso- and metafemora are dark brown to black, vein cu-a in hind wing is distinctly curved, and T1 is smooth.

\section{Host}

Unknown.

\section{Distribution in Iran}

Alborz Province (present study).

Microplitis cebes Nixon, 1970

Fig. 3

Microplitis cebes Nixon, 1970: 18, pl. 26.

Diagnosis (female)

Body length 3.0-3.8 mm; antenna as long as or slightly longer than body, F15 length $2.0 \times$ its width; anteromesoscutum rugose to rugulose; mesoscutellar disc coriaceous (Fig. 3C); vein R1 shorter than pterostigma (i.e., vein R1 longer than pterostigma half), $1^{\text {st }}$ discal cell length $1.0-1.2 \times$ its width, vein $1 \mathrm{CUb} 2.3-2.5 \times$ as long as vein $1 \mathrm{CUa}$ (Fig. 3D); T1 length $2.0 \times$ its maximum width, T1 sub-parallel- to 
parallel-sided (Fig. 3E). Colour: body black; pterostigma dark brown; legs except for metafemur and basal half of mesofemur yellow.

\section{Material examined}

IRAN - Alborz Province • 1 क ; Chalous Road, Shahrestanak; 3557'34.98" N, 51²2'20.34" E; 2305 m a.s.1.; 1 Jun. 2010; M. Khayrandish leg.; Malaise trap; TMUC-HBMMP0006. - Tehran Province • 1 \%;

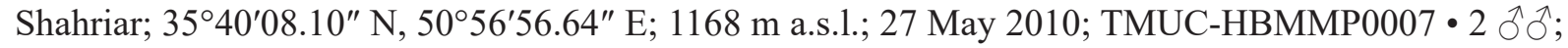
same collection data as for preceding; 15 Jun. 2010; TMUC-HBMMP0008, TMUC-HBMMP0009 - 1 , 2 ऊ; same collection data as for preceding; 20 Jun. 2010; TMUC-HBMMP0010 to TMUCHBMMP0012.

\section{Distribution in Iran}

Alborz and Tehran Provinces (present study). New record from Iran.

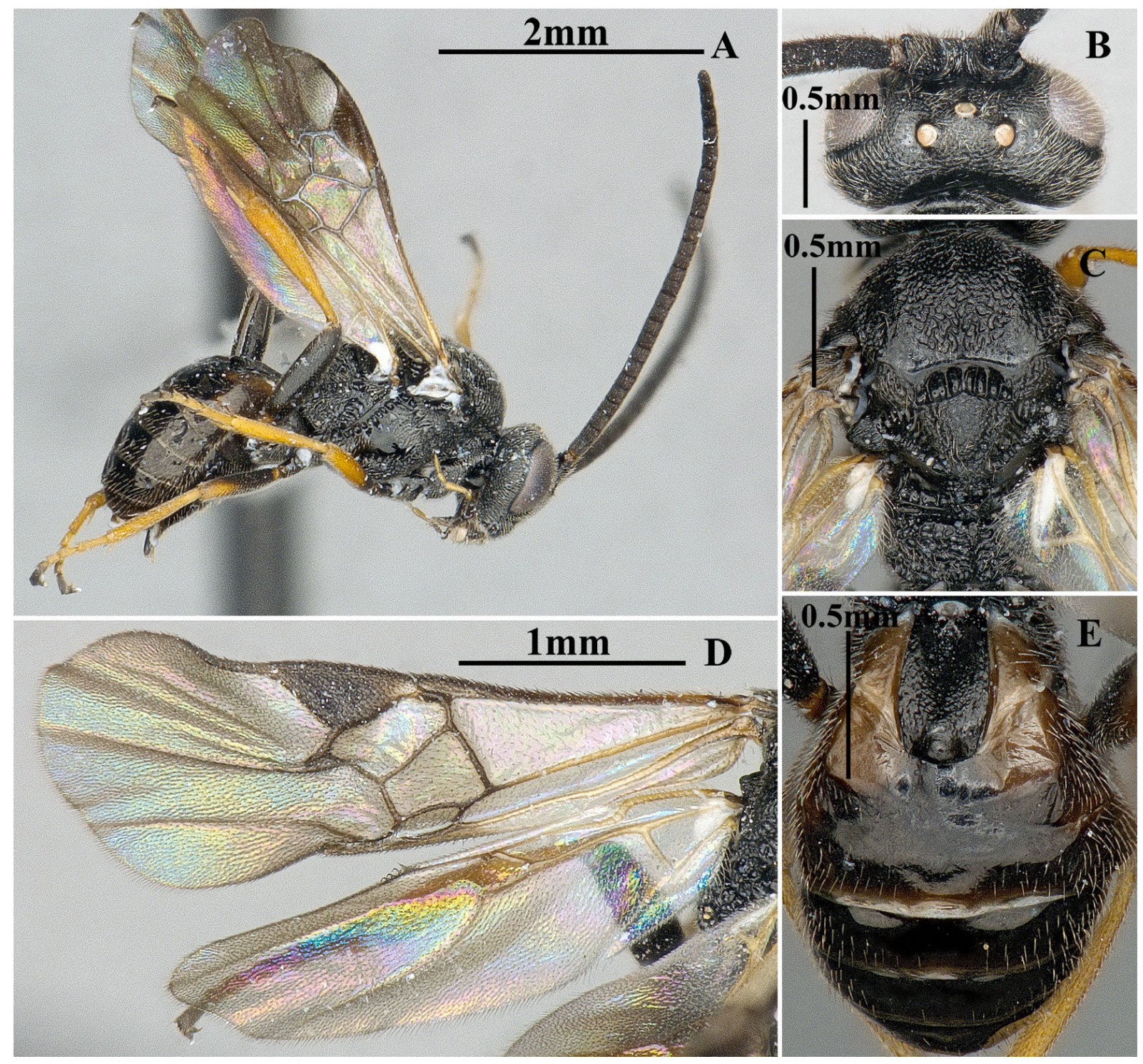

Fig. 3. Microplitis cebes Nixon, 1970, , TMUC-HBMMP0007. A. Habitus, lateral view. B. Head, dorsal view. C. Mesosoma, dorsal view. D. Wings. E. Metasoma, dorsal view. 
Microplitis decipiens Prell, 1925

Microplitis decipiens Prell, 1925: 140.

\section{Distribution in Iran}

East Azerbaijan Province (Gadallah et al. 2015).

Microplitis deprimator (Fabricius, 1798)

Ichneomon deprimator Fabricius, 1798: 227, pl. 10.

Microgaster ingratus Haliday, 1834: 236, pl. 20.

Microgaster deprimatrix Schulz, 1906: 343.

\section{Distribution in Iran}

Iran (no locality cited) (Nixon 1968).

Microplitis docilis Nixon, 1970

Fig. 4

Microplitis docilis Nixon, 1970: 28, pl. 23.

\section{Diagnosis (female)}

Body length 3.00-3.30 mm; antenna as long as or slightly longer than body, F15 length $2.0 \times$ its width (Fig. 4A); anteromesoscutum and mesoscutellar disc rugose; notauli slightly defined with coarse rugosity (Fig. 4C); vein R1 shorter than pterostigma (i.e., vein R1 longer than pterostigma half); $1^{\text {st }}$ discal cell length $1.1-1.2 \times$ its width, vein 1 CUb $2.0-2.6 \times$ as long as vein $1 \mathrm{CUa}$ (Fig. 4D); T1 length $1.5-1.6 \times$ its maximum width, more or less broadening posteriorly, T1 coarsely rugose (Fig. 4F). Colour: body black; antennal flagellum brown; scape yellow; pterostigma black or dark brown; legs yellow or reddish yellow (except for black coxae and brown or reddish brown metafemur) (Fig. 4A).

\section{Material examined}

IRAN - Qazvin Province • 3 + 9 ; Loshan; 3640'09.12" N, 49²5'37.74" E; 291 m a.s.1.; 6 Jul. 2010; M. Khayrandish leg.; Malaise trap; TMUC-HBMMP0013 to TMUC-HBMMP0015.

\section{Distribution in Iran}

Qazvin Province (present study). New record from Iran.

Microplitis eremitus Reinhard, 1880

Fig. 5

Microplitis eremita Reinhard, 1880: 360.

\section{Diagnosis (female)}

Body length 3.0-4.0 mm; antenna as long as body, F15 length $2.0 \times$ its width (Fig. $5 \mathrm{~A}$ ); anteromesoscutum rugose and rugulose; notauli well defined by rugosity; mesoscutellar disc coriaceous to rugulose (Fig. 5C); pterostigma length $2.5 \times$ its width; vein R1 shorter than pterostigma (i.e., vein R1 longer than pterostigma half); $1^{\text {st }}$ discal cell length $1.0-1.1 \times$ its width; vein 1 CUb $2.0 \times$ as long as vein $1 C U a$ (Fig. 5D); T1 length 1.5-1.6 $\times$ its maximum width, T1 more or less broadening posteriorly (Fig. 5F). 
Colour: body black; antenna, tegula and legs reddish yellow or yellow exception of dark brown metacoxa, metatibia and a dark spot at apical metafemur; pterostigma dark brown.

\section{Material examined}

IRAN - Guilan Province • 1 o; Roodsar, Rahim abad, Ghazichak; 36²45'52.62" N, 50²0'01.08" E; 1787 m a.s.l.; 17 May 2010; A. Mohammadi leg.; Malaise trap; TMUC-HBMMP0016 • 1 क ; same collection data as for preceding; 14 Jun. 2010; TMUC-HBMMP0017 • 1 \%; Roodsar, Rahim abad, Orkom; 36²5'44.34" N, 50¹8'11.88" E; 1201 m a.s.1.; 17 May 2010; A. Mohammadi leg.; Malaise trap; TMUC-HBMMP0018 • 1 क ; same collection data as for preceding; 6 Jul. 2010; TMUC-HBMMP0019 - 1 क; same collection data as for preceding; 25 Jul. 2010; TMUC-HBMMP0020. - Mazandaran

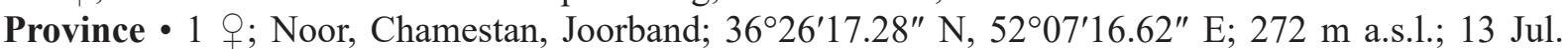
2011; A. Mohammadi leg.; Malaise trap; TMUC-HBMMP0021 • 4 우, 1 § ; same collection data as

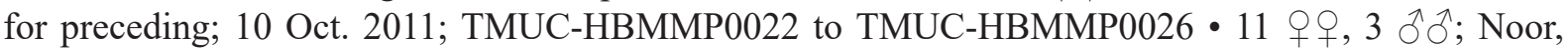
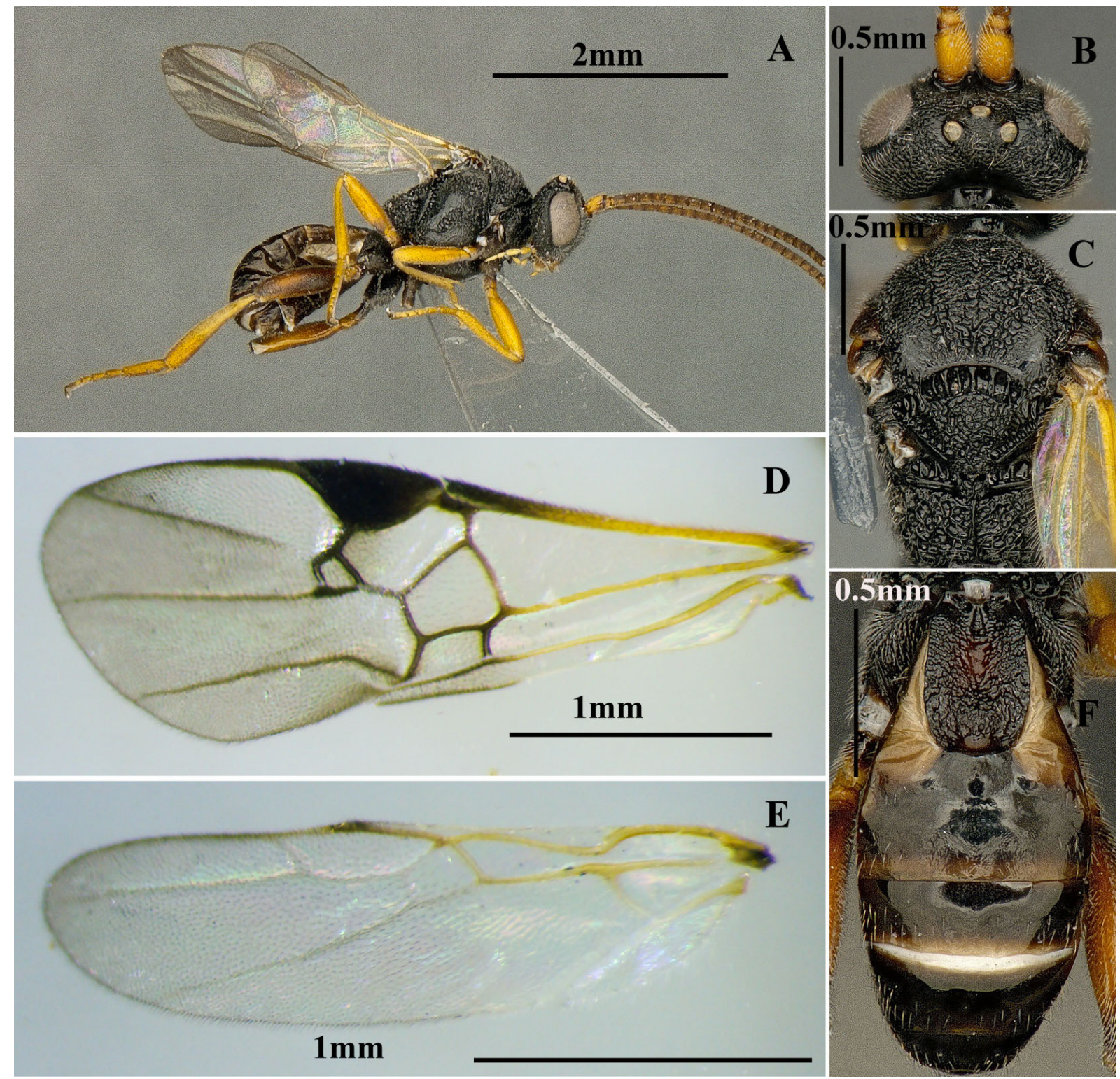

Fig. 4. Microplitis docilis Nixon, 1970,, , TMUC-HBMMP0013. A. Habitus, lateral view B. Head, dorsal view. C. Mesosoma, dorsal view. D. Fore wing. E. Hind wing. F. Metasoma, dorsal view. 
Chamestan, Tangehvaz; 36²1'55.02" N, 5206'10.74" E; 692 m a.s.1.; 26 Jul. 2011; A. Mohammadi leg.; Malaise trap; TMUC-HBMMP0027 to TMUC-HBMMP0040 • 2 q ; ; same collection data as for preceding; 10 Oct. 2011; TMUC-HBMMP0041, TMUC-HBMMP0042 2 우; same collection data as for preceding; 16 Aug. 2011; TMUC-HBMMP0043, TMUC-HBMMP0044. - Qazvin Province • 1 \&; Loshan; 36 40'09.12" N, 49²5'37.74" E; 291 m a.s.1.; 22 Jun. 2011; A. Mohammadi leg.; Malaise trap; TMUC-HBMMP0045.

\section{Distribution in Iran}

Guilan, Mazandaran and Qazvin Provinces (present study). New record from Iran.

\section{Microplitis fulvicornis (Wesmael, 1837)}

Microgaster fulvicornis Wesmael, 1837: 44.

Microgaster pallidicornis Marshall, 1898: 182, pl. 27.
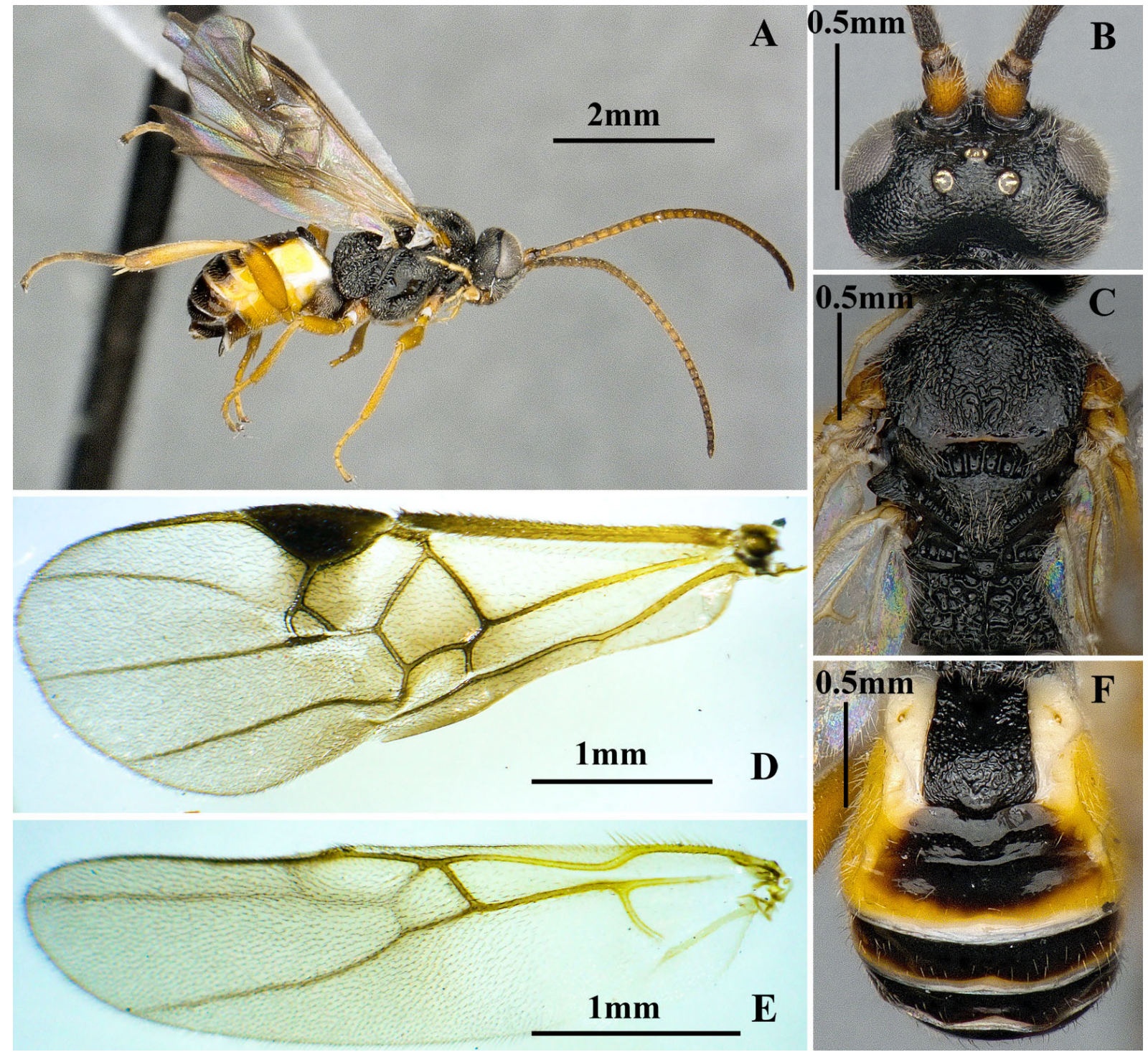

Fig. 5. Microplitis eremitus Reinhard, 1880, ๆ, TMUC-HBMMP0017. A. Habitus, lateral view. B. Head, dorsal view. C. Mesosoma, dorsal view. D. Fore wing. E. Hind wing. F. Metasoma, dorsal view. 


\section{Distribution in Iran}

Razavi Khorasan Province (Karimi-Malati et al. 2014).

\section{Microplitis kaszabi Papp, 1980}

Fig. 6

Microplitis kaszabi Papp, 1980: 406, pl. 36.

\section{Diagnosis (female)}

Body length 3.3-3.8 mm; antenna longer than body, F15 $2.0 \times$ its width (Fig. 6A); anteromesoscutum rugulose, notauli well defined by rugosity, mesoscutellar disc coriaceous (Fig. 6C); pterostigma length $2.5 \times$ its width; vein R1 shorter than pterostigma (i.e., vein R1 distinctly longer than pterostigma half);
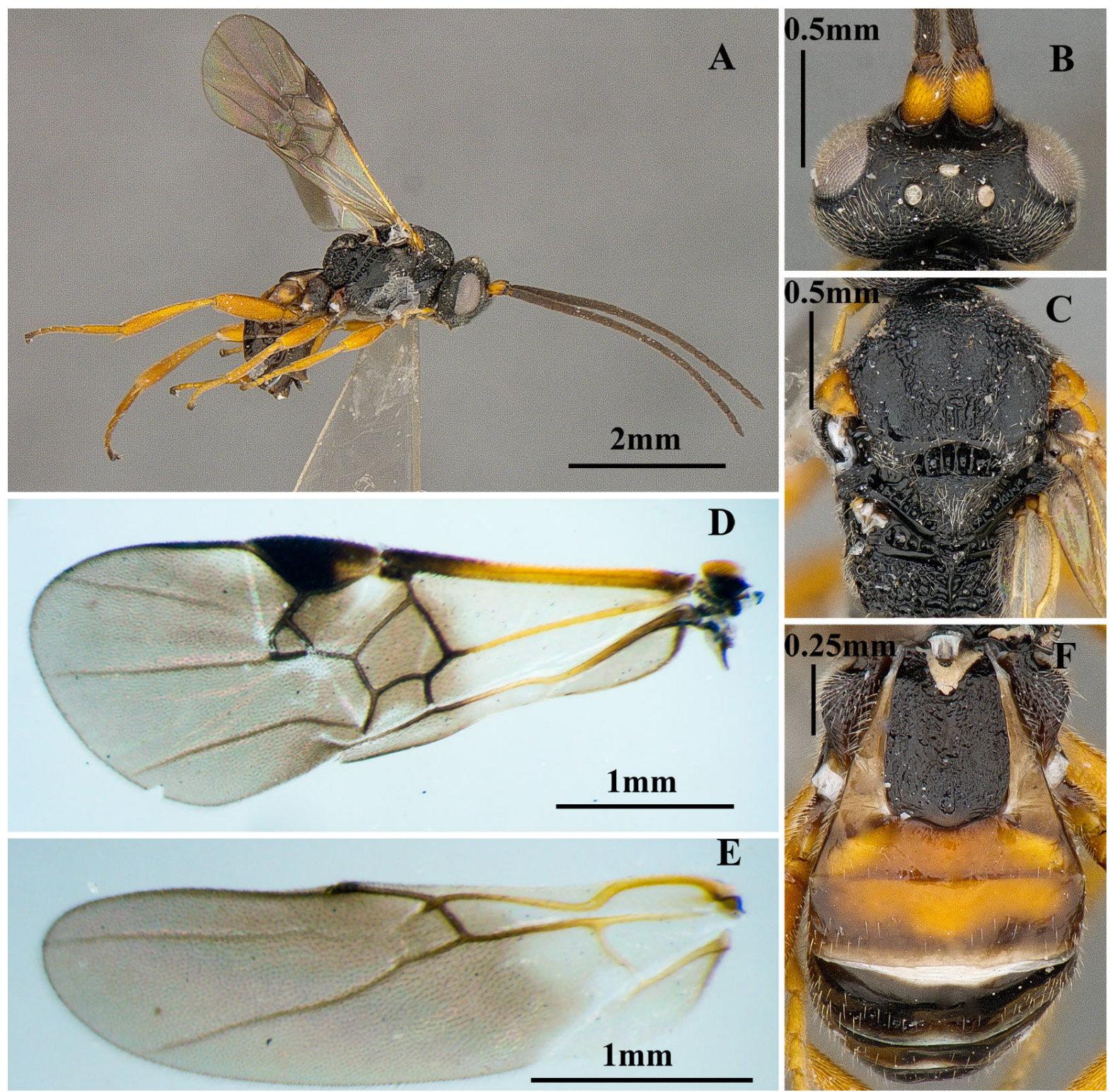

Fig. 6. Microplitis kaszabi Papp, 1980, +, TMUC-HBMMP0047. A. Habitus, lateral view. B. Head, dorsal view. C. Mesosoma, dorsal view. D. Fore wing. E. Hind wing. F. Metasoma, dorsal view. 
in $1^{\text {st }}$ discal cell length $1.2-1.3 \times$ its width; vein 1 CUb $1.7-1.8 \times$ as long as vein 1 CUa (Fig. $\left.6 \mathrm{D}\right)$; T1 rugulose, more or less broadening posteriorly and T1 length $1.5-1.6 \times$ its maximum width (Fig. 6A). Colour: body black; antenna brown; tegula, scape, T2, T3 and legs (except for black metacoxa) yellow; pterostigma dark brown to black with a small basal yellow spot.

\section{Material examined}

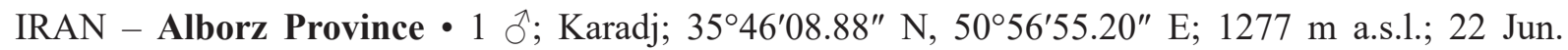
2010; A. Mohammadi leg.; Malaise trap; TMUC-HBMMP0046. - Tehran Province • 1 ; ; Shahriar; 3540'08.10" N, 5056'56.64" E; 1168 m a.s.1.; 15 Jun. 2010; A. Mohammadi leg.; Malaise trap; TMUC-

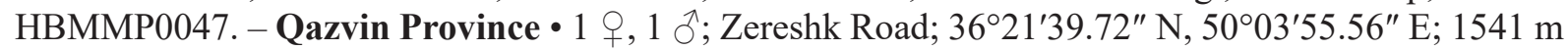
a.s.1.; 5 Jun. 2011; A. Mohammadi leg.; Malaise trap; TMUC-HBMMP0048, TMUC-HBMMP0049.

\section{Distribution in Iran}

Alborz, Tehran and Qazvin Provinces (present study). New record from Iran.

\section{Microplitis mandibularis Thomson, 1895}

Fig. 7

Microgaster (Microplitis) mandibularis Thomson, 1895: 2251, pl. 4.

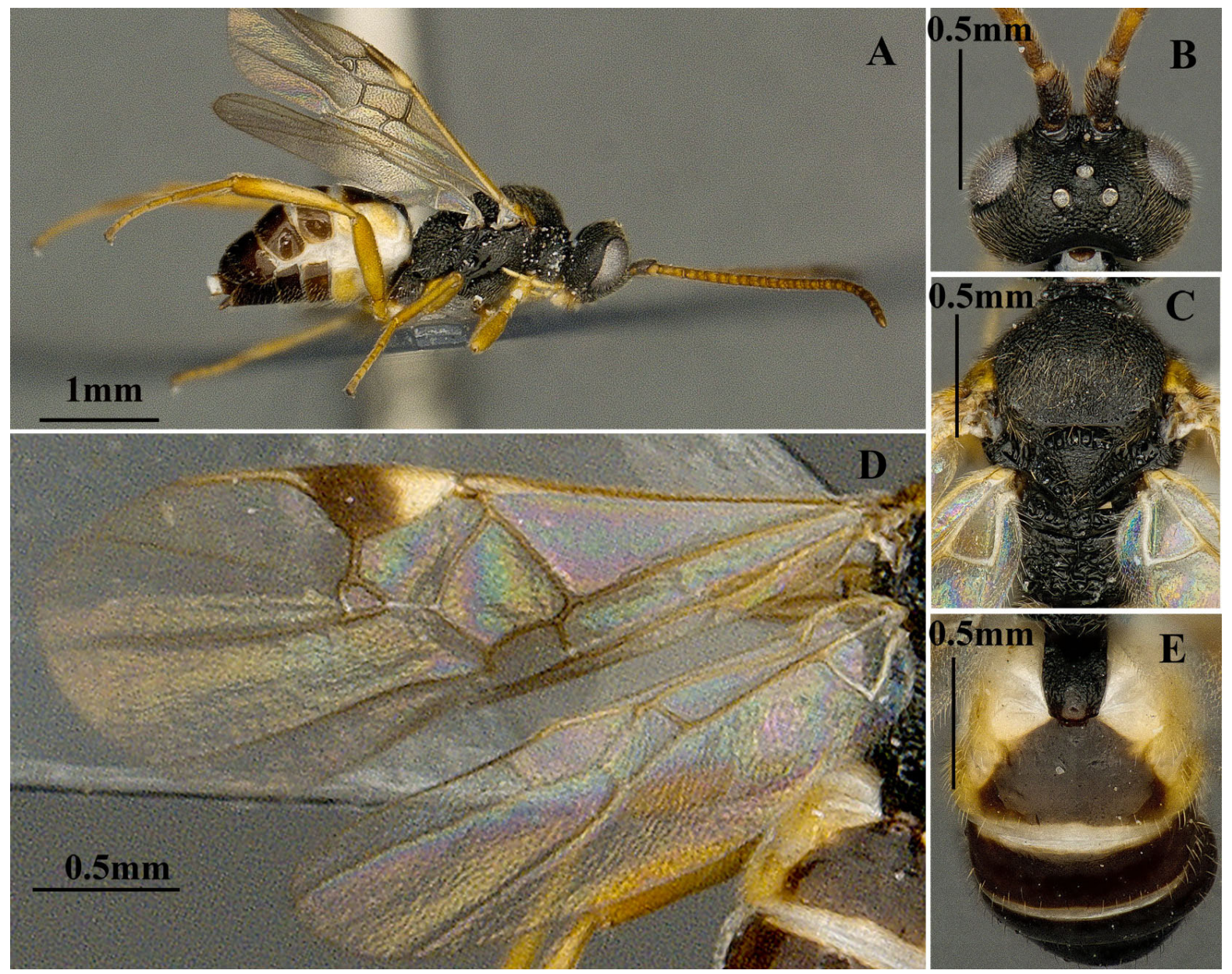

Fig. 7. Microplitis mandibularis Thomson, 1895, †, TMUC-HBMMP0051. A. Habitus, dorsal view. B. Head, dorsal view. C. Mesosoma, dorsal view. D. Wings. E. Metasoma, dorsal view. 


\section{Diagnosis (female)}

Body length 3.0-3.5 mm; antenna as long as body, F15 length $2.0 \times$ its width (Fig. 7A); in dorsal view, head width less than $2.0 \times$ its length (Fig. 7B); anteromesoscutum and mesoscutellar disc coriaceous (Fig. 7C); pterostigma length $2.5 \times$ its width; vein R1 longer than pterostigma half; $1^{\text {st }}$ discal cell length $1.2-1.3 \times$ its width; vein 1 CUb $3.0 \times$ as long as vein $1 \mathrm{CUa}$ (Fig. 7D); T1 length $2.5 \times$ its maximum width, T1 rugulose and parallel-sided (Fig. 7E). Colour: body black; antenna and tegula reddish yellow; basal one third of pterostigma and legs yellow (except for black metacoxa).

\section{Material examined}

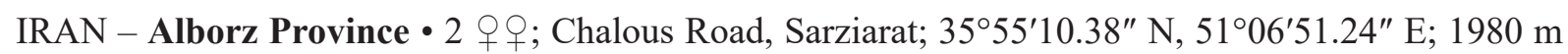
a.s.1.; 20 Jul. 2010; M. Khayrandish leg.; Malaise trap; TMUC-HBMMP0050, TMUC-HBMMP0051 •

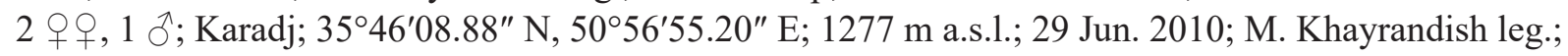
Malaise trap; TMUC-HBMMP0052 to TMUC-HBMMP0054.

\section{Distribution in Iran}

Fars Province (Samin et al. 2018), Alborz Province (present study).

Microplitis marshallii Kokujev, 1898

Microplitis marshallii Kokujev, 1898 (in Marshall 1900): 183, pl. 9.

\section{Distribution in Iran}

Ardabil Province (Gadallah et al. 2015).

Microplitis mediator (Haliday, 1834)

Microgaster mediator Haliday, 1834: 235.

Microgaster medianus Ruthe, 1860: 127.

Microplitis halidayi Fahringer, 1937: 285.

Microplitis pseudomedianus Fahringer, 1937: 267.

\section{Distribution in Iran}

No locality cited (Belokobylskij et al. 2019).

Microplitis ochraceus Szepligeti, 1896

Fig. 8

Microplitis ochraceus Szepligeti, 1896: 307.

Microplitis flaviventris Ivanov, 1898 (in Ivanov 1899): 342.

\section{Diagnosis (female)}

Body length 3.5-4.0 mm; antenna as long as body, F15 length $2.0 \times$ its width (Fig. 8A); anteromesoscutum and mesoscutellar disc weakly rugulose or coriaceous (Fig. 8C); pterostigma length $2.1 \times$ its width; vein $\mathrm{R} 1$ longer than pterostigma half; $1^{\text {st }}$ discal cell length $1.3-1.4 \times$ its width; vein 1 CUb $2.0 \times$ as long as vein 1 CUa (Fig. 8D); T1 rugose, distinctly broadening posteriorly and T1 length slightly longer than its maximum width; T2 rugulose (Fig. 8D). Colour: body yellow or reddish yellow except for black head, brown mesoscutellar disc and metanotum; basal one third of pterostigma yellow. 


\section{Material examined}

IRAN - Tehran Province • 1 q; Shahriar; 3540'08.10" N, 5056'56.64" E; 1168 m a.s.1.; 15 Jun. 2010; M. Khayrandish leg.; Malaise trap; TMUC-HBMMP0055.

\section{Distribution in Iran}

Ardabil Province (Telenga 1955), Iran (locality not exactly defined) (Papp 1984; Tobias 1986), Golestan Province (Ghahari et al. 2011d), Alborz and Tehran Provinces (Farahani et al. 2014c; present study).



Fig. 8. Microplitis ochraceus Szepligeti, 1896, +, TMUC-HBMMP0055. A. Habitus, lateral view. B. Head, dorsal view. C. Mesosoma, dorsal view. D. Fore wing. E. Hind wing. F. Metasoma, dorsal view. 
Microplitis pallidipennis Tobias, 1964

Fig. 9

Microplitis pallidipennis Tobias, 1964: 213, pl. 50.

\section{Diagnosis (female)}

Body length $4.2 \mathrm{~mm}$; antenna as long as body, F15 length $2.0 \times$ as long as its width; vertex punctate; notauli well defined with crowded rugosity; lateral and median lobe of anteromesoscutum weakly punctate and shiny; mesoscutellar disc anteriorly or antero-medially weakly punctate, shiny and its

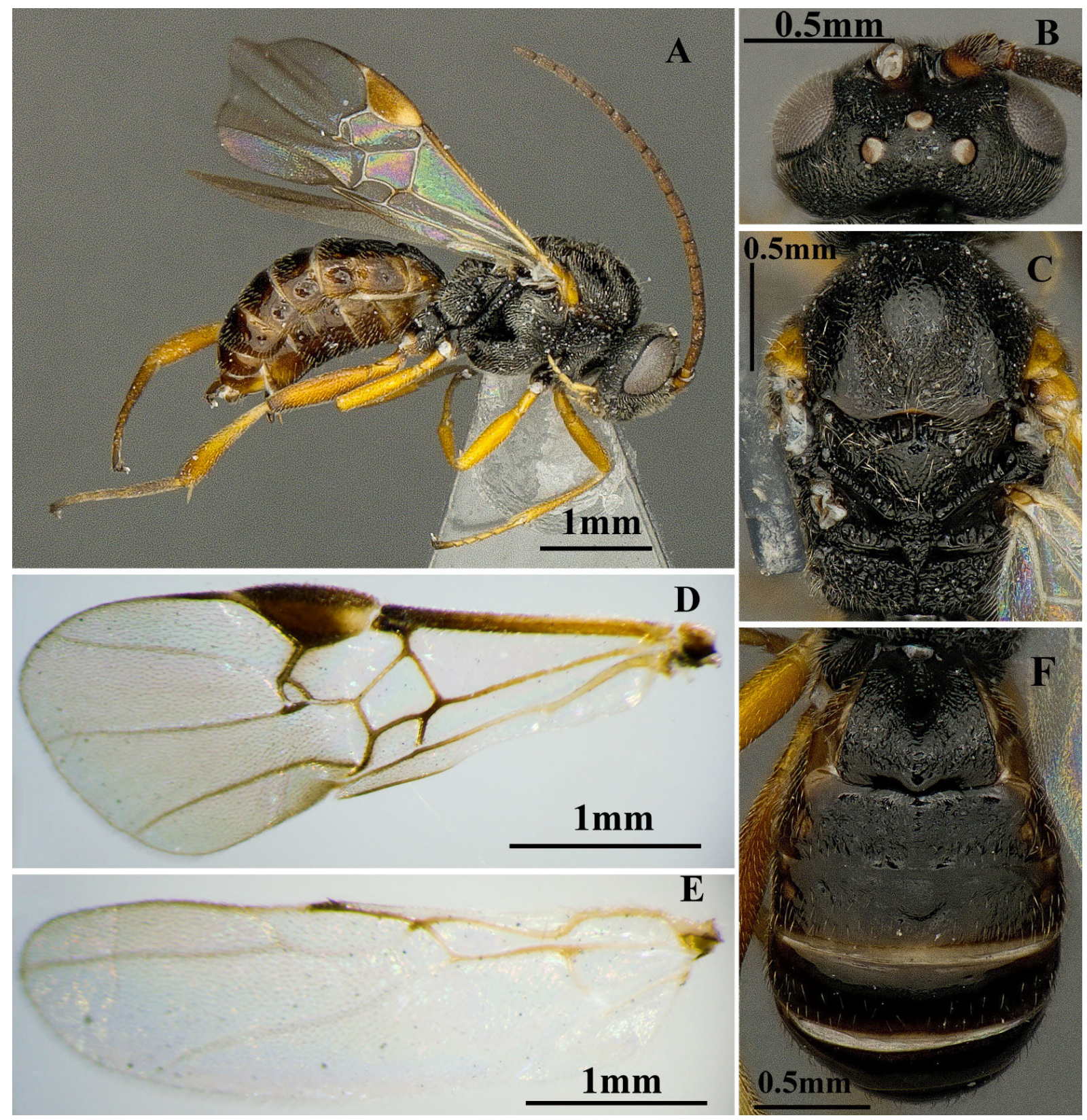

Fig. 9. Microplitis pallidipennis Tobias, 1964, + , TMUC-HBMMP0056. A. Habitus, lateral view. B. Head, dorsal view. C. Mesosoma, dorsal view. D. Fore wing. E. Hind wing. F. Metasoma, dorsal view. 
posterior area coriaceous (Fig. 9C); pterostigma length 2.1-2.2 $\times$ its width; vein R1 as long as pterostigma half; $1^{\text {st }}$ discal cell length $1.3 \times$ its width; vein 1 CUb $2.0 \times$ as long as vein 1 CUa (Fig. 9D); T1 rugulose, distinctly broadening posteriorly, its length equal or slightly longer than its maximum width; T2 weakly wrinkled; T3 and the following tergites smooth (Fig. 9F). Colour: body black; antenna brown; tegula and legs reddish yellow (except for black coxae); basal one quarter of pterostigma with a yellow spot.

\section{Material examined}

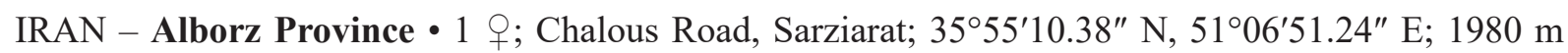
a.s.l.; 15 Jun. 2010; M. Khayrandish leg.; Malaise trap; TMUC-HBMMP0056.

\section{Distribution in Iran}

Alborz Province (present study). New record from Iran.

\section{Microplitis rufiventris Kokujev, 1914}

Fig. 10

Microplitis rufiventris Kokujev, 1914: 513.

\section{Diagnosis (female)}

Body length 2.8-3.5 mm; antenna as long as body, F15 length $2.0 \times$ its width (Fig. 10A); in dorsal view, head width $1.8 \times$ its length (Fig. 10B); anteromesoscutum rugulose; mesoscutellar disc weakly rugulose (Fig. 10C); vein R1 as long as pterostigma half; $1^{\text {st }}$ discal cell length $1.2-1.3 \times$ its width; vein $1 \mathrm{CUb} 2.0 \times$ as long as vein $1 \mathrm{CUa}$ (Fig. 10D); T1 length $2.0 \times$ its maximum width, T1 smooth, shiny and parallel- to sub-parallel-sided (Fig. 10F). Colour: body black; antenna and metatarsus brown; tegula, tergites and legs reddish yellow to yellow; basal one third of pterostigma with a yellow spot (Fig. 10).

\section{Material examined}

IRAN - Alborz Province • 2 क 9 ; Karadj; 3546'08.88" N, 5056'55.20" E; 1277 m a.s.1.; 29 Jun. 2010; M. Khayrandish leg.; Malaise trap; TMUC-HBMMP0057, TMUC-HBMMP0058.

\section{Distribution in Iran}

Alborz Province (Farahani et al. 2014c; present study), Tehran Province (Farahani et al. 2014c), Qazvin Province (Farahani et al. 2016).

Microplitis scrophulariae Szepligeti, 1898

Microplitis scrophulariae Szepligeti, 1898: 385.

\section{Distribution in Iran}

Kashan Province (Ghahari et al. 2011c); Iran (no locality cited) (Tobias 1976).

\section{Microplitis sofron Nixon, 1970}

Microplitis sofron Nixon, 1970: 8, pl. 10.

\section{Distribution in Iran}

Qazvin Province (Samin et al. 2019). 
Microplitis spectabilis (Haliday, 1834)

Fig. 11

Microgaster spectabilis Haliday, 1834: 236, pl. 4.

Microgaster fossulatus Bouche, 1834: 161.

Microgaster parvulus Ruthe, 1860: 139.

Microgaster seuratii Marshall, 1998: 370.

Dapsilotoma testaceipes Cameron, 1906: 101.

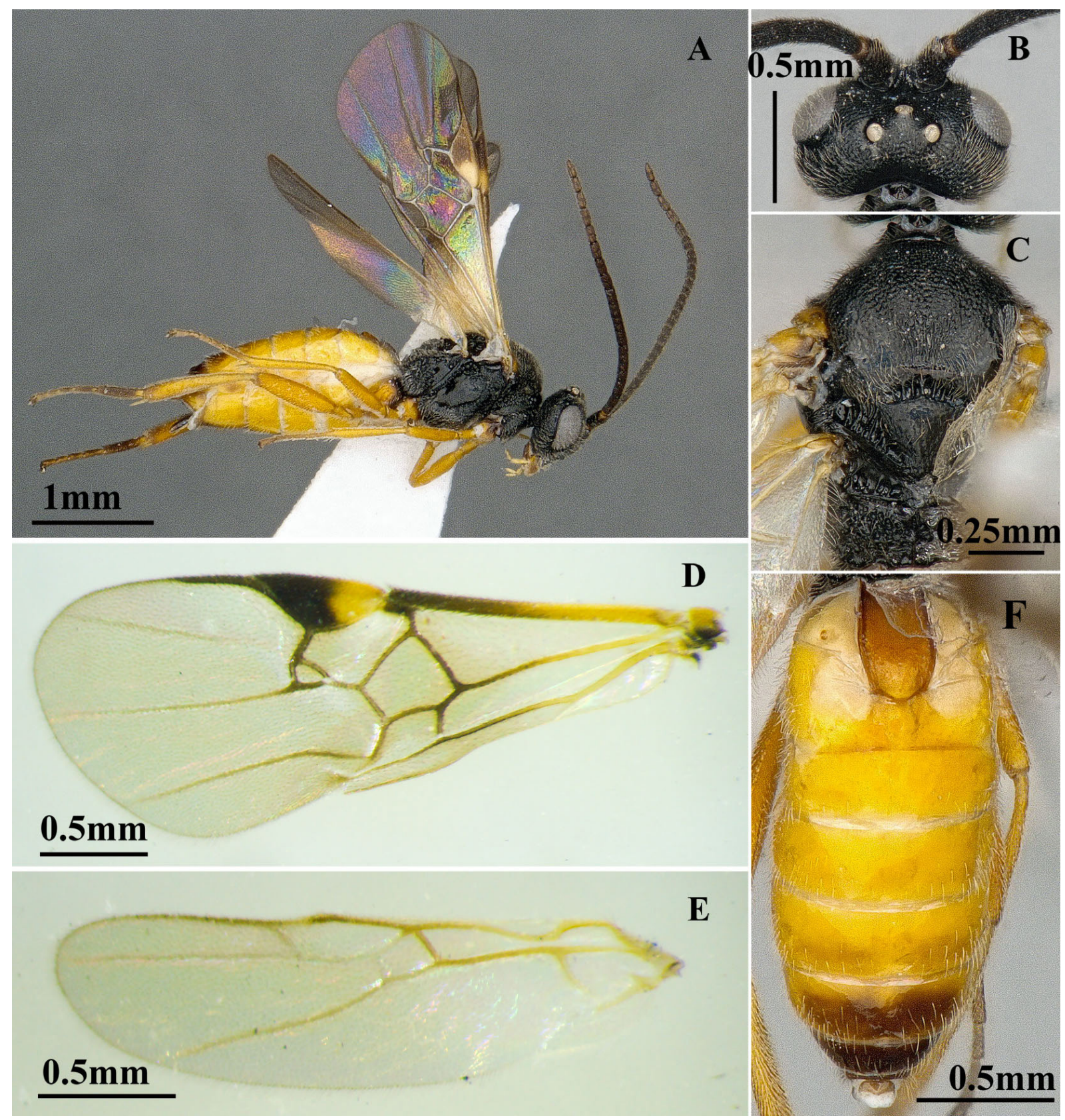

Fig. 10. Microplitis rufiventris Kokujev, 1914, +, TMUC-HBMMP0057. A. Habitus, lateral view. B. Head, dorsal view. C. Mesosoma, dorsal view. D. Fore wing. E. Hind wing. F. Metasoma, dorsal view. 


\section{Diagnosis (female)}

Body length 2.4-3.0 mm; antenna shorter than body, F15 length $2.0 \times$ its width (Fig. 11A); head in dorsal view less transverse and head width $1.7 \times$ its length (Fig. 11B); anteromesoscutum rugulose; notauli slightly defined by rugosity; mesoscutellar disc coriaceous (Fig. 11C); pterostigma length $2.5 \times$ its width; $1^{\text {st }}$ discal cell length $1.20-1.4 \times$ its width; vein $1 \mathrm{CUb} 1.7-1.9 \times$ as long as vein $1 \mathrm{CUa}$; vein R1 slightly longer than pterostigma half (Fig. 11D); vein cu-a in hind wing slightly curved (Fig. 11E); T1 length $1.5 \times$ its maximum width, T1 more or less broadening posteriorly, rugose to rugulose. Colour: body black; antenna brown; basal one third of pterostigma with a yellow spot; legs yellow, except for black metacoxa, metafemur with a variable coloured pattern (reddish brown, brown to black).

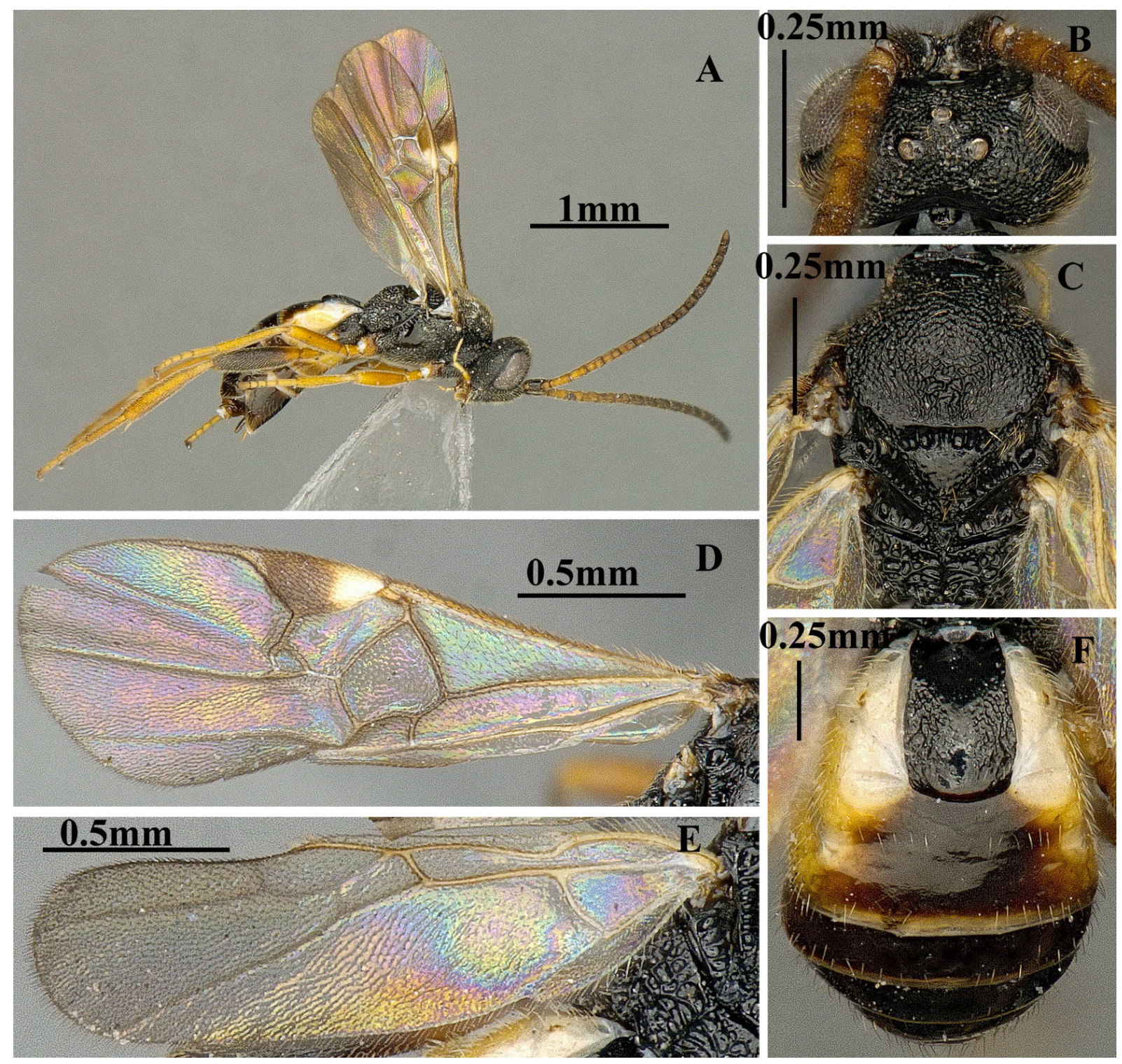

Fig. 11. Microplitis spectabilis (Haliday, 1834), +, TMUC-HBMMP0061. A. Habitus, lateral view. B. Head, dorsal view. C. Mesosoma, dorsal view. D. Fore wing. E. Hind wing. F. Metasoma, dorsal view. 


\section{Material examined}

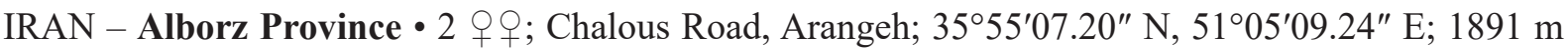
a.s.1.; 29 Jun. 2010; M. Khayrandish leg.; Malaise trap; TMUC-HBMMP0059, TMUC-HBMMP0060 - 1 क ; same collection data as for preceding; 14 Jul. 2010; TMUC-HBMMP0061 • 1 q, 1 ₹; same collection data as for preceding; 16 Aug. 2010; TMUC-HBMMP0062, TMUC-HBMMP0063・11 $q$; same collection data as for preceding; 14 Sep. 2010; TMUC-HBMMP0064 to TMUC-HBMMP0074 - $4 \widehat{\partial} \widehat{\widehat{\partial}}$; same collection data as for preceding; 5 Oct. 2010; TMUC-HBMMP0075 to TMUC-

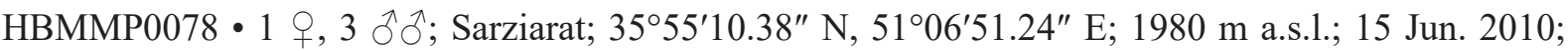

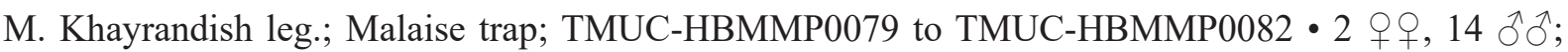
same collection data as for preceding; 6 Jul. 2010; TMUC-HBMMP0083 to TMUC-HBMMP0098 • 3 웅, 5 ठึ; ; same collection data as for preceding; 14 Jul. 2010; TMUC-HBMMP099 to TMUC-

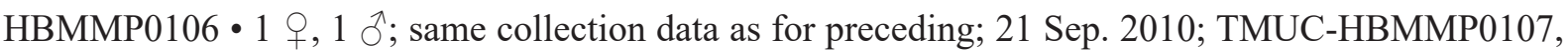
TMUC-HBMMP0108. - Guilan Province • 1 क , 1 万'; Roodsar, Rahim abad, Ghazichak; 36 45'52.62" N, 50²0'01.08" E; 1787 m a.s.1.; 7 Jun. 2010; M. Khayrandish leg.; Malaise trap; TMUC-HBMMP0109, TMUC-HBMMP0110 • 1 ; ; same collection data as for preceding; 11 Jul. 2010; TMUC-HBMMP0111 - 1 +, 7 ठ̊j; same collection data as for preceding; 20 Sep. 2010; TMUC-HBMMP0112 to TMUCHBMMP0119・1 +, 3 ๙ ; same collection data as for preceding; 22 Jun. 2010; TMUC-HBMMP0120

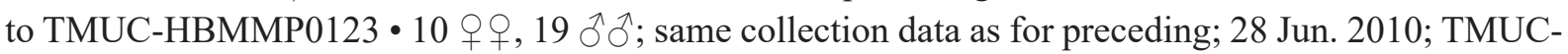
HBMMP0124 to TMUC-HBMMP0152 • 5 우, 18 ふぇ ; same collection data as for preceding; 5 Sep. 2010; TMUC-HBMMP0153 to TMUC-HBMMP0175• 8 우, 1 \%; same collection data as for preceding;

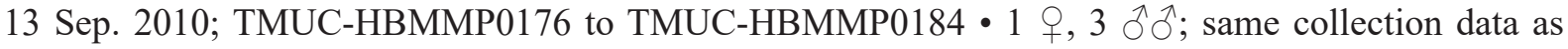
for preceding; 26 Sep. 2010; TMUC-HBMMP0185 to TMUC-HBMMP0188 • 1 đో; Roodsar, Rahim abad, Orkom; 36²4'44.34" N, 50¹8'11.88" E; 1201 m a.s.1.; 17 May 2010; M. Khayrandish leg.;

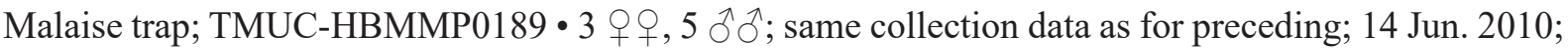

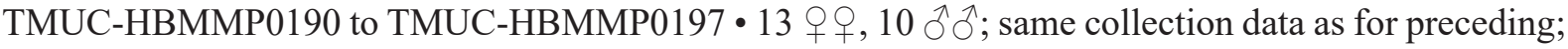

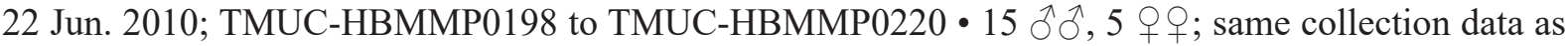
for preceding; 6 Jul. 2010; TMUC-HBMMP0221 to TMUC-HBMMP0240 • 2 우, 1 §ో; same collection data as for preceding; 23 Aug. 2010; TMUC-HBMMP0241 to TMUC-HBMMP0243 • 2 q $Q$; same collection data as for preceding; 5 Sep. 2010; TMUC-HBMMP0244, TMUC-HBMMP0245 • 1 § ; same collection data as for preceding; 19 Sep. 2010; TMUC-HBMMP0246・4 $q$; ; same collection data as

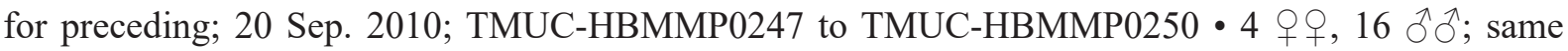
collection data as for preceding; 26 Sep. 2010; TMUC-HBMMP0251 to TMUC-HBMMP0270・11 $ᄋ$,

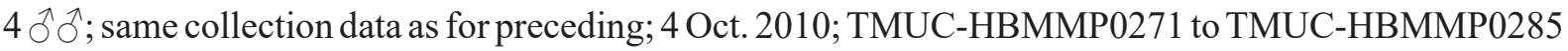

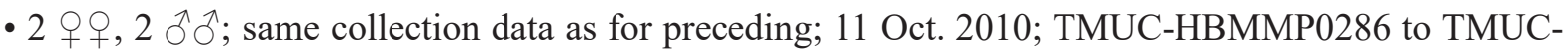
HBMMP0289 • 1 †, 1 đ; same collection data as for preceding; 18 Oct. 2010; TMUC-HBMMP0290, TMUC-HBMMP0291 • 1 \%; same collection data as for preceding; 19 Oct. 2010; TMUC-HBMMP0292 - 1 क; same collection data as for preceding; 1 Nov. 2010; TMUC-HBMMP0293 • 1 +, 1 ○; Roodsar, Rahim abad, Ziaz; 3652'27.18" N, 50¹3'24.78” E; 490 m a.s.1.; 14 Oct. 2010; M. Khayrandish leg.; Malaise trap; TMUC-HBMMP0294, TMUC-HBMMP0295 • 2 우, 2 ○ึ; same collection data as for preceding; 17 Jun. 2010; TMUC-HBMMP0296 to TMUC-HBMMP0299 • 1 क; same collection

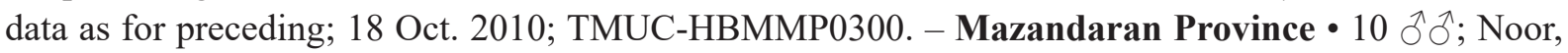
Chamestan, Tangehvaz; 36² $21^{\prime} 55.68^{\prime \prime}$ N, 52 $06^{\prime} 10.32^{\prime \prime}$ E; $702 \mathrm{~m}$ a.s.1.; 5 Sep. 2011, M. Khayrandish leg.; Malaise trap; TMUC-HBMMP0301 to TMUC-HBMMP0310 • 3 우, $3 \hat{\jmath} \delta^{\lambda}$; same collection data as for preceding; 26 Sep. 2011; TMUC-HBMMP0311 to TMUC-HBMMP0316. - Qazvin Province • 1 q, 1 ð’; Zereshk Road; 36²1'43.02" N, 5003'53.22" E; 1553 m a.s.1.; 9 Jun. 2011; M. Khayrandish leg.; Malaise trap; TMUC-HBMMP0317, TMUC-HBMMP0318. 


\section{Distribution in Iran}

Isfahan (Ghahari et al. 2011c), East Azarbaijan (Rastegar et al. 2012), Alborz, Mazandaran, Guilan and Qazvin Provinces (present study).

Microplitis spinolae (Nees, 1834)

Fig. 12

Microgaster spinolae Nees, 1834: 166, pl. 4.

Microplitis sapporoensis Ashmead, 1906: 194.

Microplitis radiorimata Telenga, 1955: 142, pl. 34.
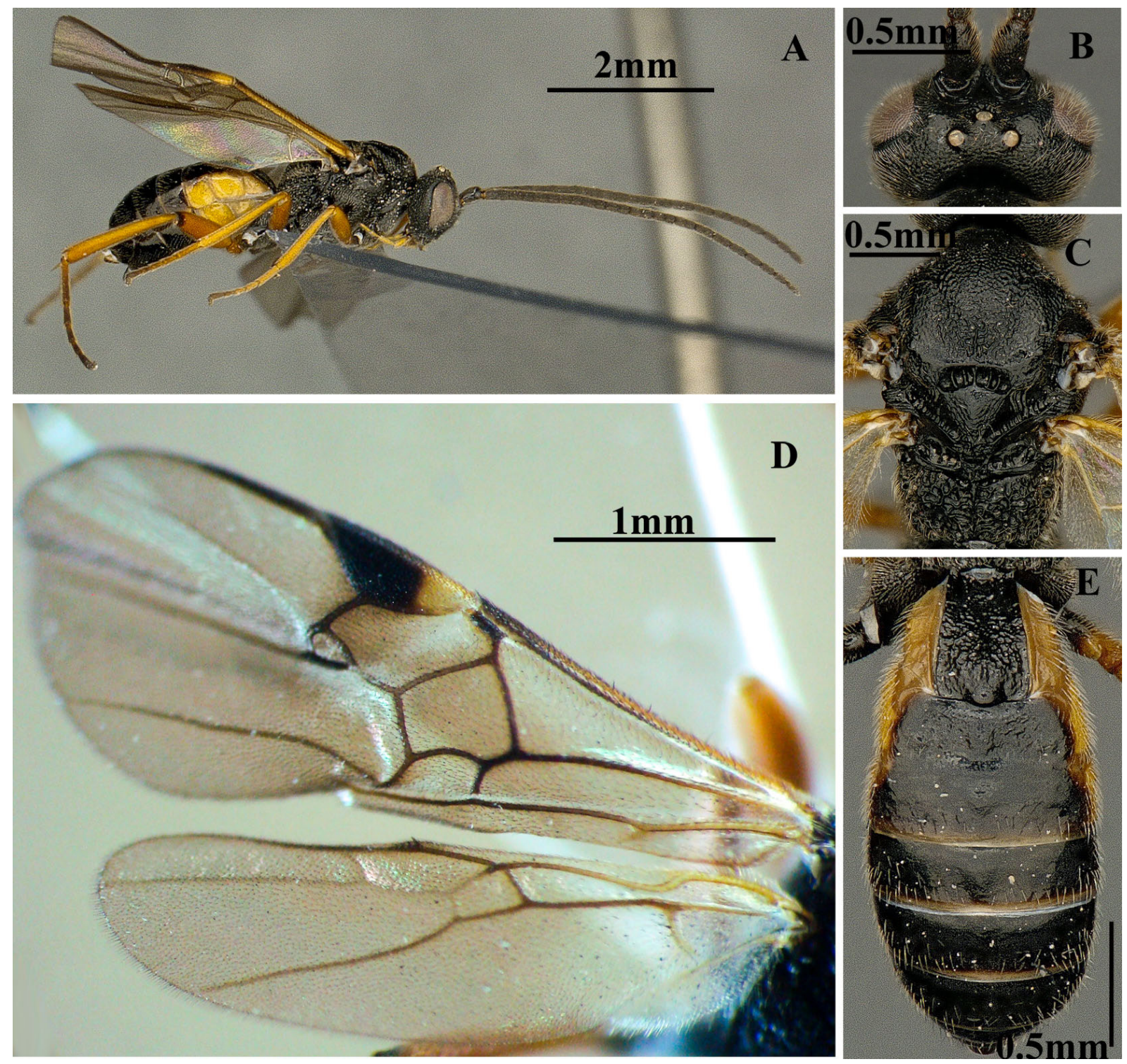

Fig. 12. Microplitis spinolae (Nees, 1834), $q$, TMUC-HBMMP0321. A. Habitus, lateral view. B. Head, dorsal view. C. Mesosoma, dorsal view. D. Wings. E. metasoma, dorsal view. 


\section{Diagnosis (female)}

Body length 3.5-4.5 mm; antenna as long as body, F15 length $2.0 \times$ its width (Fig. 12A), above of antennal socket (on upper face) polished, while in other species is completely sculptured (Fig. 12B); anteromesoscutum and mesoscutellar disc rugulose; notauli slightly defined by rugosity (Fig. 12C); pterostigma length $2.2-2.4 \times$ its width; $1^{\text {st }}$ discal cell length $1.2-1.4 \times$ its width; vein 1 CUb $1.9-2.0 \times$ as long as vein 1CUa; vein R1 longer than pterostigma half (Fig. 12D); T1 length 1.4-1.6 $\times$ its maximum width; T1 rugulose, more or less broadening posteriorly; T2 weakly wrinkled (Fig. 12E). Colour: body black; basal one third of pterostigma with a yellow spot; legs reddish yellow, except for black coxae, metafemur with a black spot apically.

\section{Material examined}

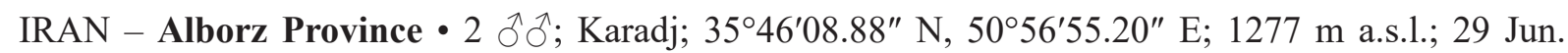
2010; A. Nadimi leg.; Malaise trap; TMUC-HBMMP0319, TMUC-HBMMP0320. - Guilan Province

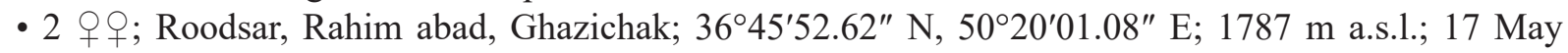
2010; M. Khayrandish leg.; Malaise trap; TMUC-HBMMP0321, TMUC-HBMMP0322 • 1 क; Orkom; 36 $6^{\circ} 5^{\prime} 44.34^{\prime \prime} \mathrm{N}, 50^{\circ} 18^{\prime} 11.88^{\prime \prime}$ E; $1201 \mathrm{~m}$ a.s.1.; 6 Jul. 2010; A. Nadimi leg.; Malaise trap; TMUCHBMMP0323 • 2 우; Ziaz; 3652'27.18" N, 50¹3'24.78” E; 490 m a.s.1.; 24 May 2010; A. Nadimi leg.; Malaise trap; TMUC-HBMMP0324, TMUC-HBMMP0325 • 1 q; same collection data as for preceding; 7 Jun. 2010; TMUC-HBMMP0326• 1 ; ; same collection data as for preceding; 6 Jun. 2010;

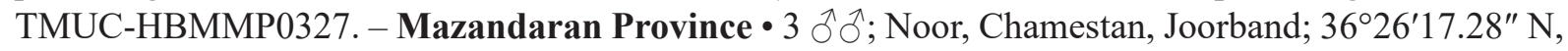
52 07'16.62" E; 272 m a.s.1.; 5 Sep. 2011; A. Nadimi leg.; Malaise trap; TMUC-HBMMP0328, TMUCHBMMP0330. - Tehran Province • 1 q; Shahriar; 3540'08.10" N, 5056'56.64" E; 1168 m a.s.1.; 28 Sep. 2010; A. Nadimi leg.; Malaise trap; TMUC-HBMMP0331.

\section{Distribution in Iran}

Iran (locality not exactly defined) (Nixon 1970); Alborz, Guilan, Mazandaran and Qazvin Provinces (present study).

\section{Microplitis tuberculifer (Wesmael, 1837)}

Fig. 13

Microgaster tuberculifer Wesmael, 1837: 43.

Microgaster calcaratus Thomson, 1895: 2249, pl. 11.

Microgaster trochanterata Thomson, 1895: 2249, pl. 25.

Microplitis manevali Gautier \& Bonnamour, 1939: 190, pl. 4.

\section{Diagnosis (female)}

Body length 3.0-3.8 mm; antenna as long as body, F15 length 2.0-3.0 $\times$ its width (Fig. 13A); in dorsal view, head width $1.8 \times$ its length; vertex punctate; anteromesoscutum and mesoscutellar disc coriaceous (Fig. 13C); pterostigma length $2.3-2.5 \times$ its width; $1^{\text {st }}$ discal cell length $1.2-1.3 \times$ its width; vein $1 \mathrm{CUb} 2.0 \times$ as long as vein 1CUa; vein R1 longer than pterostigma half (Fig. 13D); T1 length $2.5 \times$ its maximum width, T1 rugose or rugulose and parallel-sided (Fig. 13F). Colour: body black; antenna brown or black; tegula brownish yellow; pterostigma dark brown with a basal yellow spot; legs yellow, except for brownish yellow metatarsus; T2 and T3 variably coloured, yellow, brown or black.

\section{Material examined}

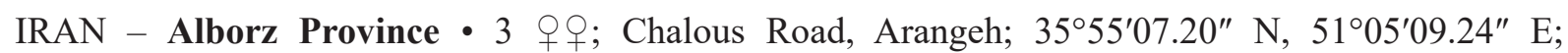
1891 m a.s.1.; 22 Jun. 2010; M. Khayrandish leg.; Malaise trap; TMUC-HBMMP0332 to TMUC- 
HBMMP0334 - 3 + $O$; same collection data as for preceding; 14 Jul. 2010; TMUC-HBMMP0335 to

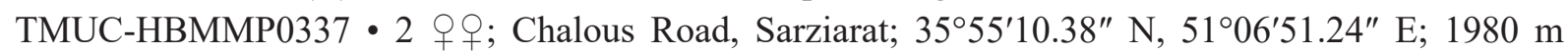
a.s.1.; 8 Jun. 2010; M. Khayrandish leg.; Malaise trap; TMUC-HBMMP0338, TMUC-HBMMP0339

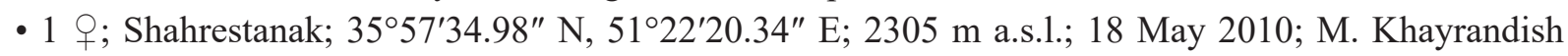
leg.; Malaise trap; TMUC-HBMMP0340 • 1 क; same collection data as for preceding; 5 Jul. 2010; TMUC-HBMMP0341 • 15 우, 1 §;; same collection data as for preceding; 13 Jul. 2010; TMUC-

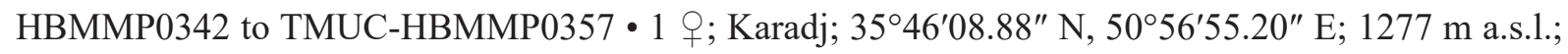
15 Jun. 2010; M. Khayrandish leg.; Malaise trap; TMUC-HBMMP0358. - Guilan Province $\bullet 2$ 우;
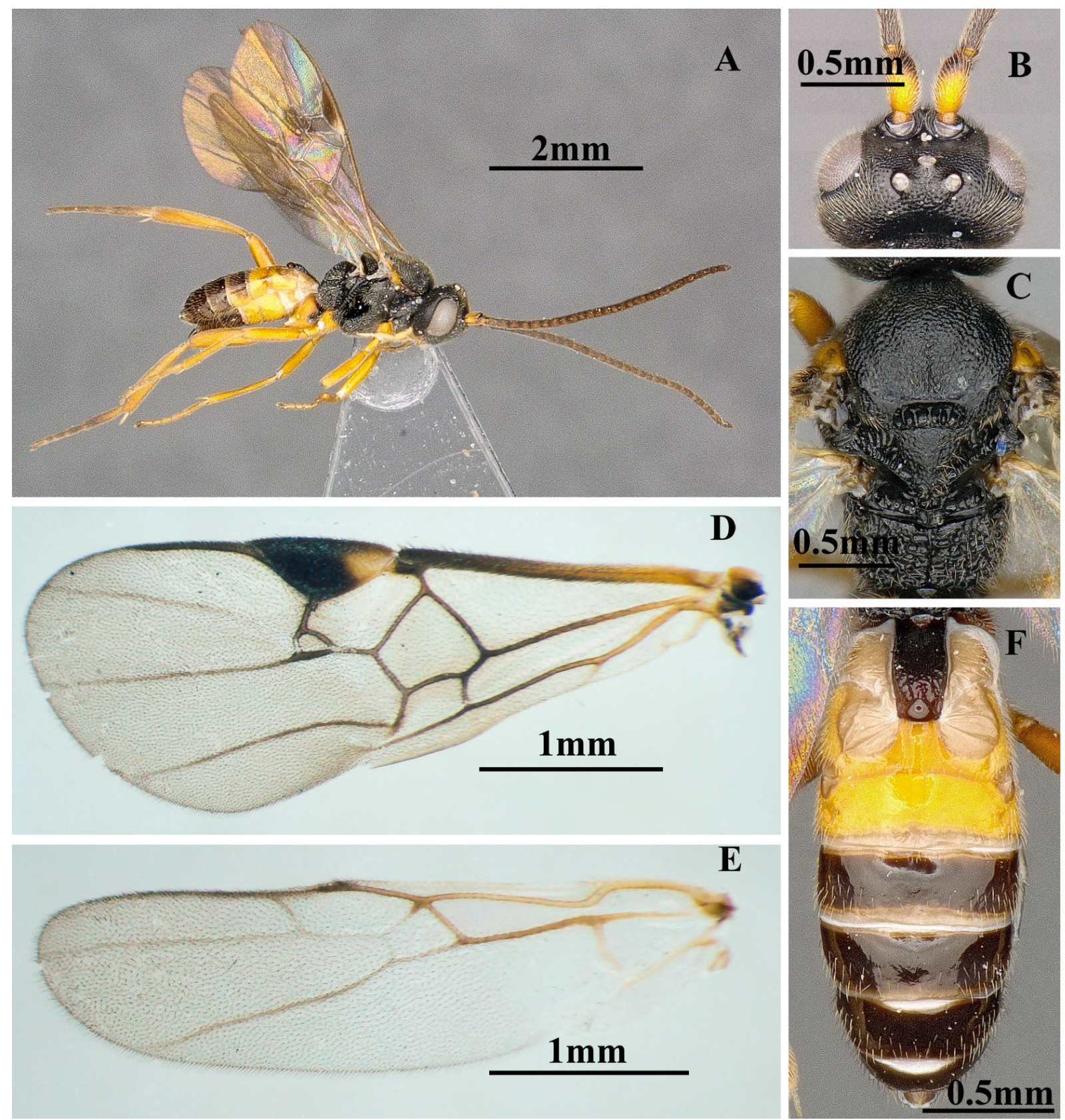

Fig. 13. Microplitis tuberculifer (Wesmael, 1837),, , TMUC-HBMMP0335. A. Habitus, lateral view. B. Fore wing. C. Hind wing. D. Head, dorsal view. E. Mesosoma, dorsal view. F. Metasoma, dorsal view. 
Astaneh Ashrafiyeh, Eshman kamachal; 37²2'03.66" N, 4957'57.84" E; 1 m b.s.1.; 10 May 2010; M. Khayrandish leg.; Malaise trap; TMUC-HBMMP0359, TMUC-HBMMP0360 • 1 क; Roodsar, Ziaz; 36 52'34.44" N, 50¹3'17.40" E; 537 m a.s.l.; 12 Apr. 2010; M. Khayrandish leg.; Malaise trap; TMUC-HBMMP0361 • 1 \%; same collection data as for preceding; 25 Apr. 2010; TMUC-HBMMP0362 - 5 우; same collection data as for preceding; 10 May 2010; TMUC-HBMMP0363 to TMUCHBMMP0367 7 q $\circ$; same collection data as for preceding; 17 May 2010; TMUC-HBMMP0368 to TMUC-HBMMP0374 • 2 우, 3 ๙ึ; same collection data as for preceding; 20 May 2010; TMUCHBMMP0375 to TMUC-HBMMP0379 • 2 우; same collection data as for preceding; 24 May 2010;

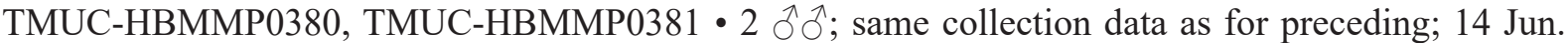
2010; TMUC-HBMMP0382, TMUC-HBMMP0383 • 2 우; same collection data as for preceding;

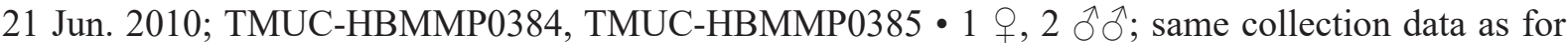
preceding; 22 Jun. 2010; TMUC-HBMMP0386 to TMUC-HBMMP0388 • 1 q; same collection data as for preceding; 6 Jul. 2010; TMUC-HBMMP0389 - 1 సं; same collection data as for preceding; $15 \mathrm{Jul}$. 2010; TMUC-HBMMP0390 • 2 오우 Roodsar, Ghazichak; 36 45'52.62" N, 50²0'01.08" E; $1787 \mathrm{~m}$ a.s.1.; 3 May 2010; M. Khayrandish leg.; Malaise trap; TMUC-HBMMP0391, TMUC-HBMMP0392 • 2 + $O$; same collection data as for preceding; 10 May 2010; TMUC-HBMMP0393, TMUC-HBMMP0394 - 3 우, 1 ơ; same collection data as for preceding; 17 May 2010; TMUC-HBMMP0395 to TMUCHBMMP0398 • 1 क; same collection data as for preceding; 31 May 2010; TMUC-HBMMP0399 •

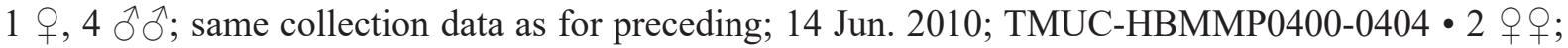
same collection data as for preceding; 6 Jul. 2010; TMUC-HBMMP0405, TMUC-HBMMP0406 • 2 우우, 8 ते; same collection data as for preceding; 11 Jul. 2010; TMUC-HBMMP0407 to TMUC-

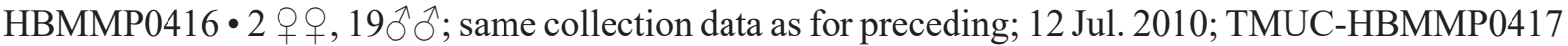
to TMUC-HBMMP0437 • 2 우; Roodsar, Orkom; 36²45'44.34" N, 50¹8'11.88" E; $1201 \mathrm{~m}$ a.s.1.; 6 Apr. 2010; M. Khayrandish leg.; Malaise trap; TMUC-HBMMP0438, TMUC-HBMMP0439 • 1 ○; same collection data as for preceding; 12 Apr. 2010; TMUC-HBMMP0440 • 1 \%; same collection data as for preceding; 26 Apr. 2010; TMUC-HBMMP0441 • 1 क; same collection data as for preceding; 10 May 2010; TMUC-HBMMP0442 • 4 o ; ; same collection data as for preceding; 17 May 2010; TMUC-HBMMP0443 to TMUC-HBMMP0446 3 q 9 ; same collection data as for preceding; 27 May 2010; TMUC-HBMMP0447 to TMUC-HBMMP0449・2 $\widehat{\jmath}$; ; same collection data as for preceding; 7 Jun. 2010; TMUC-HBMMP0450, TMUC-HBMMP0451 1 क ; same collection data as for preceding; 14 Jun. 2010; TMUC-HBMMP0452 • 1 क ; same collection data as for preceding; 6 Jul. 2010; TMUCHBMMP0453 • 1 क; same collection data as for preceding; 19 Jul. 2010; TMUC-HBMMP0454 - 1 \%; same collection data as for preceding; $21 \mathrm{Jul}$. 2010; TMUC-HBMMP0455 • 1 q; same collection data

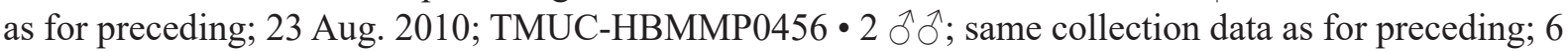
Sep. 2010; TMUC-HBMMP0457, TMUC-HBMMP0458 • 1 q; same collection data as for preceding;

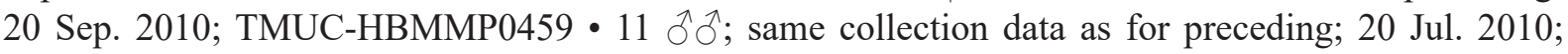
TMUC-HBMMP0460 to TMUC-HBMMP0470. - Mazandaran Province • 2 $q$ q ; Noor, Chamestan, Gaznasara; $36^{\circ} 16^{\prime} 56.82^{\prime \prime}$ N, 52 ${ }^{\circ} 10^{\prime} 58.50^{\prime \prime}$ E; 2032 m a.s.1.; 28 Jun. 2011; M. Khayrandish leg.; Malaise trap; TMUC-HBMMP0471, TMUC-HBMMP0472 • 1 क; same collection data as for preceding; $13 \mathrm{Jul}$. 2011; TMUC-HBMMP0473 • 1 q ; Noor, Chamestan, Tangehvaz; 36 21' $55.68^{\prime \prime}$ N, 52 $2^{\circ} 06^{\prime} 10.32^{\prime \prime}$ E; $702 \mathrm{~m}$ a.s.1.; 26 May 2011; M. Khayrandish leg.; Malaise trap; TMUC-HBMMP0474 • 1 q; same collection data as for preceding; $30 \mathrm{Jul}$. 2011; TMUC-HBMMP0475 • 1 \%; same collection data as for preceding; 5 Aug. 2011; TMUC-HBMMP0476 • 1 +, 1 त; same collection data as for preceding; 16 Aug. 2011; TMUC-HBMMP0477, TMUC-HBMMP0478 - 2 q $q$; same collection data as for preceding; 20 Sep. 2011; TMUC-HBMMP0479, TMUC-HBMMP0480 • 2 우, 1 ㄱ; same collection data as for preceding; 6 Oct. 2011; TMUC-HBMMP0481 to TMUC-HBMMP0483・ 1 \%; same collection data as for preceding; 10 Oct. 2011; TMUC-HBMMP0484 • 1 q; same collection data as for preceding; 28 Oct. 2011; TMUCHBMMP0485 - 2 우; Noor, Faculty of Natural Resources and Marine Sciences; 36 34'52.98" N, 52 $2^{\circ} 2^{\prime} 45.78^{\prime \prime}$ E; 14 m b.s.1.; 25 May 2011; M. Khayrandish leg.; Malaise trap; TMUC-HBMMP0486, TMUC-HBMMP0487 • 1 क; same collection data as for preceding; 7 Jun. 2011; TMUC-HBMMP0488 
- 2 우, 1 今ं; same collection data as for preceding; 30 Jun. 2011; TMUC-HBMMP0489 to TMUCHBMMP0491 • 1 q; same collection data as for preceding; 28 Jul. 2011; TMUC-HBMMP0492 • 1 ; ; same collection data as for preceding; 10 Aug. 2011; TMUC-HBMMP0493 • 2 우; Noor, Chamestan, Joorband; 36²6'15.54" N, 5207'13.50" E; 275 m a.s.l.; 8 Oct. 2011; Khayrandish leg.; Malaise trap; TMUC-HBMMP0494, TMUC-HBMMP0495. - Tehran Province • 1 o; Shahriar; 3540'08.10" N, 5056'56.64" E; 1168 m a.s.1.; 8 Jun. 2010; Khayrandish leg.; Malaise trap; TMUC-HBMMP0496 • 1 + ; same collection data as for preceding; 30 Jun. 2010; TMUC-HBMMP0497 • 1 त; same collection data as for preceding; 29 Sep. 2010; TMUC-HBMMP0498.

\section{Distribution in Iran}

Ilam Province (Ghahari et al. 2011a), Alborz, Guilan, Mazandaran and Tehran Provinces (present study).

Microplitis varipes (Ruthe, 1860)

Fig. 14

Microgaster varipes Ruthe, 1860: 136.
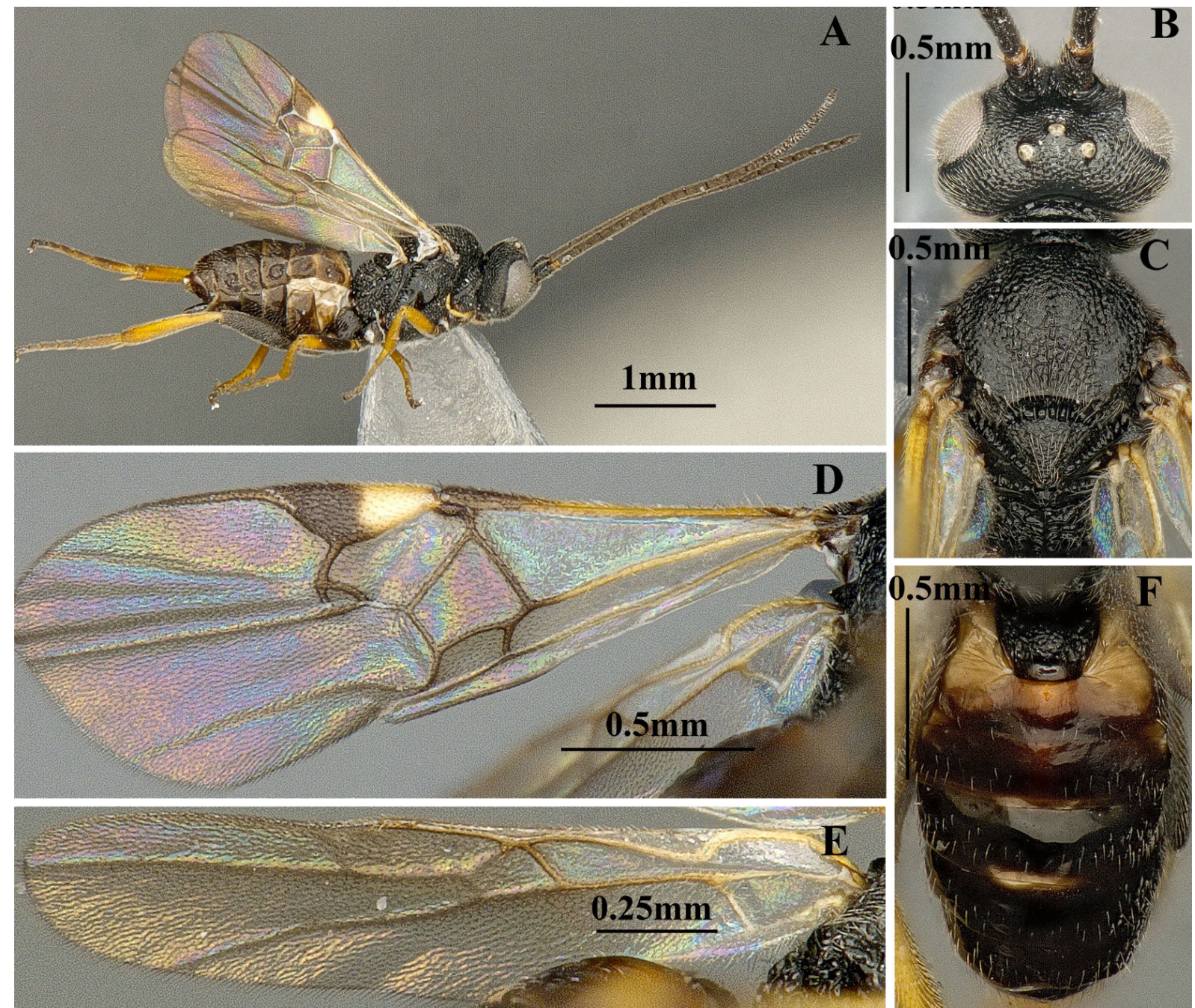

Fig. 14. Microplitis varipes (Ruthe, 1860), $q$, TMUC-HBMMP0512. A. Habitus, lateral view. B. Fore wing. C. Hind wing. D. Head, dorsal view. E. Mesosoma, dorsal view. F. Metasoma, dorsal view. 
Diagnosis (female)

Body length 2.8-3.5 mm; antenna longer than body, F15 length $2.0 \times$ its width (Fig. 14A); anteromesoscutum and mesoscutellar disc densely rugulose (Fig. 14C) and notauli slightly defined by rugosity; $1^{\text {st }}$ discal cell length $1.3-1.4 \times$ its width; vein $1 \mathrm{CUb} 2.0-2.2 \times$ as long as vein 1 CUa; vein R1 longer than pterostigma half (Fig. 14D); T1 length $1.6 \times$ its maximum width, T1 rugulose, sub-parallelsided and rounded apically (Fig. 14F). Colour: body black; basal one third of pterostigma with a yellow spot; legs reddish or brownish yellow, except for black metafemur and a black spot at basal half of mesofemur.

\section{Material examined}

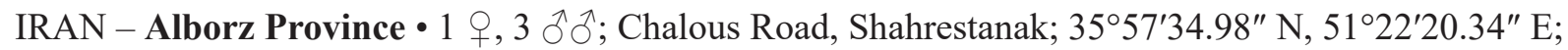
2305 m a.s.l.; 6 Jul. 2010; Khayrandish leg.; Malaise trap; TMUC-HBMMP0499 to TMUC-HBMMP0502

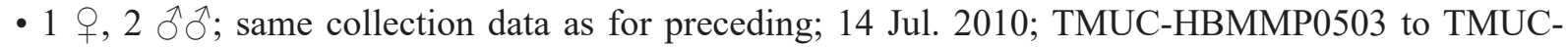

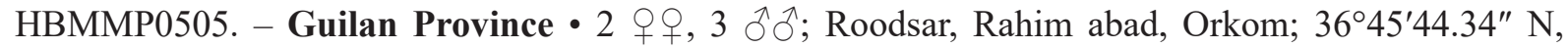
50¹8'11.88" E; 120 m a.s.l.; 19 Jul. 2010; Khayrandish leg.; Malaise trap; TMUC-HBMMP0506 to TMUC-HBMMP0510 • 1 क; same collection data as for preceding; 6 Jul. 2010; TMUC-HBMMP0511 - 10 우; Ghazichak; 36 $45^{\prime} 52.62^{\prime \prime}$ N, 50²0'01.08" E; 1787 m a.s.1.; 11 Jul. 2010; Khayrandish leg.; Malaise trap; TMUC-HBMMP0512 to TMUC-HBMMP0521. - Qazvin Province • 3 $q$; Zereshk Road; $36^{\circ} 25^{\prime} 23.88^{\prime \prime} \mathrm{N}, 50^{\circ} 06^{\prime} 37.68^{\prime \prime} \mathrm{E}$; 1926 m a.s.1.; 6 Jul. 2011; TMUC-HBMMP0522 to TMUCHBMMP0524 - 2 우; same collection data as for preceding; 17 Aug. 2011; TMUC-HBMMP0525, TMUC-HBMMP0526 • 1 क ; same collection data as for preceding; 27 Sep. 2011; TMUC-HBMMP0527 - 2 우우 Z Zereshk Road; 36²1'39.72" N, 5003'55.56" E; 1541 m a.s.1.; 25 May 2011; Khayrandish leg.; Malaise trap; TMUC-HBMMP0528, TMUC-HBMMP0529. - Tehran Province • 1 q; Peykanshahr, Iran National Botanical Garden; 3544'19.91" N, 51¹0'52.49" E; 1265 m a.s.1.; 14 May 2010; TMUCHBMMP0530.

\section{Distribution in Iran}

Alborz, Guilan, Tehran and Qazvin Provinces (present study). New record from Iran.

Microplitis viduus (Ruthe, 1860)

Microgaster viduus Ruthe, 1860: 134.

\section{Distribution in Iran}

Qazvin Province (Ghahari et al. 2011b).

Microplitis xanthopus (Ruthe, 1860)

Microgaster xanthopus Ruthe, 1860: 147.

Microgaster tenuipes Thomson, 1895: 2246.

\section{Distribution in Iran}

No locality cited (Belokobylskij et al. 2019).

\section{Discussion}

The present study is the first faunistic overview of the genus Microplitis of Iran, in which we present a key for the identification of all known Iranian species. Our samples were collected from five provinces of northern Iran including Alborz, Qazvin, Guilan, Tehran and Mazandaran. The sampled regions in 
Table 1. Updated list of the species of Microplitis Förster, 1862 (Hymenoptera: Braconidae, Microgasterinae) recorded from Iran.

\begin{tabular}{|c|c|}
\hline Parasitoid species & References \\
\hline Microplitis aduncus (Ruthe, 1860) & Papp (1984) \\
\hline Microplitis albipennis Abdinbekova, 1969 & Samin et al. (2018) \\
\hline Microplitis alborziensis Abdoli \& Talebi sp. nov. & present study \\
\hline Microplitis cebes Nixon, 1970 & present study \\
\hline Microplitis decipiens Prell, 1925 & Gadallah et al. (2015) \\
\hline Microplitis deprimator (Fabricius, 1798) & Nixon (1968) \\
\hline Microplitis docilis Nixon, 1970 & present study \\
\hline Microplitis eremitus Reinhard, 1880 & present study \\
\hline Microplitis fulvicornis (Wesmael, 1837) & Karimi-Malati et al. (2014) \\
\hline Microplitis kaszabi Papp, 1980 & present study \\
\hline Microplitis mandibularis Thomson, 1895 & Samin et al. (2018), present study \\
\hline Microplitis marshallii Kokujev, 1898 & Gadallah et al. (2015) \\
\hline Microplitis mediator (Haliday, 1834) & Belokobylskij et al. (2019) \\
\hline Microplitis ochraceus Szepligeti, 1896 & Farahani et al. (2014c), present study \\
\hline Microplitis pallidipennis Tobias, 1964 & present study \\
\hline Microplitis rufiventris Kokujev, 1914 & Farahani et al. (2014c), present study \\
\hline Microplitis scrophulariae Szepligeti, 1898 & Ghahari et al. (2011c) \\
\hline Microplitis sofron Nixon, 1970 & Samin et al. (2019) \\
\hline Microplitis spectabilis (Haliday, 1834) & Ghahari et al. (2011b), present study \\
\hline Microplitis spinolae (Nees, 1834) & Nixon (1970), present study \\
\hline Microplitis tuberculifer (Wesmael, 1837) & Ghahari et al. (2011a), present study \\
\hline Microplitis varipes (Ruthe, 1860) & present study \\
\hline Microplitis viduus (Ruthe, 1860) & Ghahari et al. (2011b) \\
\hline Microplitis xanthopus (Ruthe, 1860) & Belokobylskij et al. (2019) \\
\hline
\end{tabular}

this study belong to the Irano-Anatolian and Caucasus hotspots, which are expected to have the highest biodiversity in Iran (Kiani et al. 2017). According to previous studies, 17 species of Microplitis had been reported from Iran (Farahani et al. 2016; Samin et al. 2018; Fernandez-Triana et al. 2020). In the present study, thirteen species were collected and identified, including six new records for the Iranian fauna and one new species. Two species, M. kaszabi and M. pallidipennis, are new records for the west Palaearctic region. The updated checklist of Microplitis from Iran now consists of 24 species (Table 1).

In the present study, Malaise traps were set up in protected areas in five provinces and collection was made during the same period uniformly (from March to November of 2010 and 2011). During the collection period, two Malaise traps in Ziaz (Gilan Province) and Tangevaz (Mazandaran Province) were damaged by wild animals, and replaced by new ones. The Alborz Mountains separate the tropical Caspian Sea area (Gilan and Mazandaran Provinces) from Alborz, Tehran and Qazvin Provinces. Gilan and Mazandaran Provinces are located on the northern slopes of the Alborz Mountains with humid subtropical climate, annual rainfall of about $1500 \mathrm{~mm}$, moderate temperature and high relative humidity leading to diverse vegetation. Alborz, Tehran and Qazvin Provinces are located on the southern slopes of Alborz Mountains with semi-arid steppe climate and annual rainfall of about $300 \mathrm{~mm}$. A total of 290 specimens of the genus Microplitis were collected in northern Iran, representing 13 species. The 


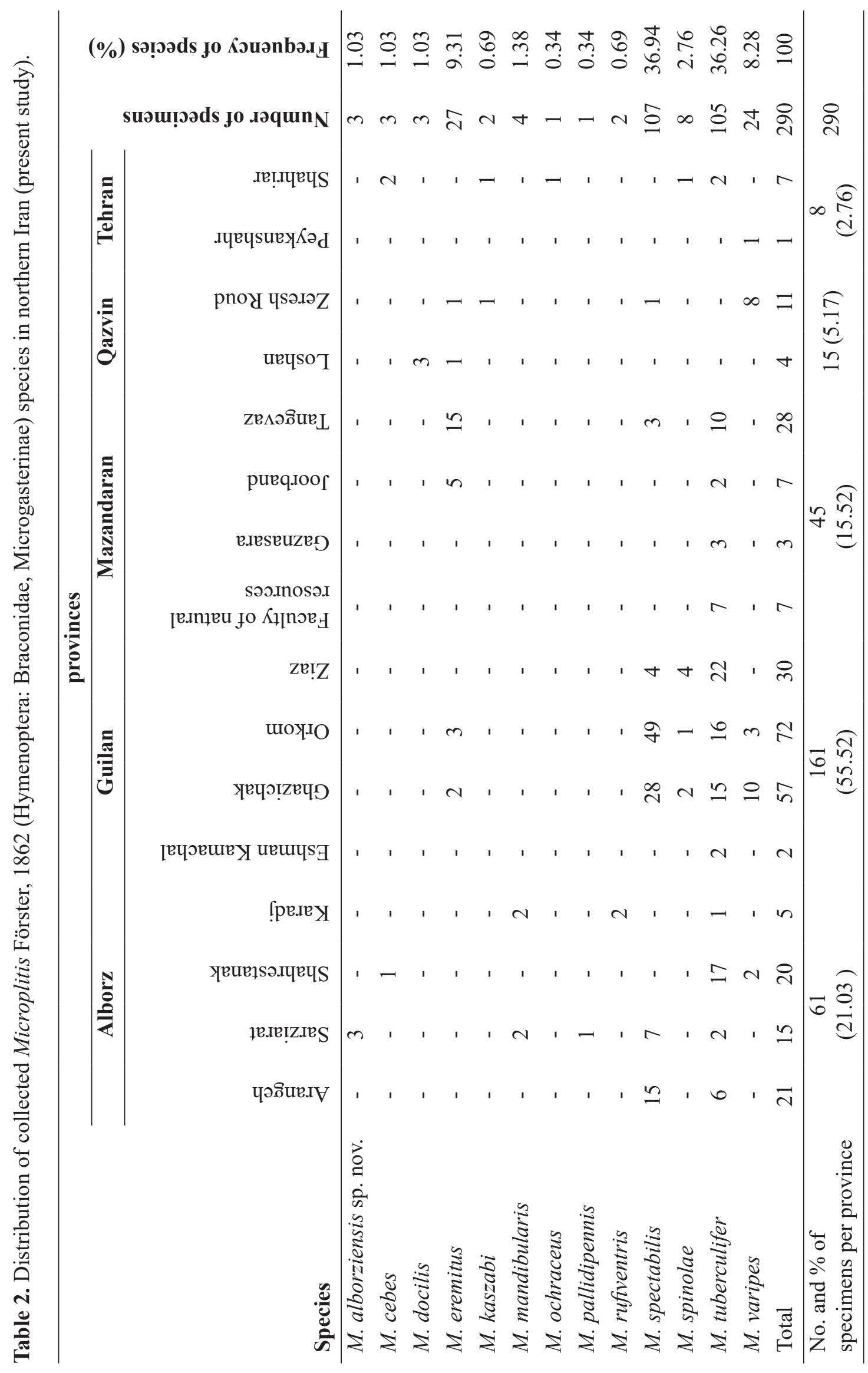


collection data set was showing a huge variation in the number of specimens per site (ranging from one to 72 specimens). Two hundred six specimens (71.03\%) were collected from the northern slopes of the Alborz Mountains. The largest number of specimens was found in Orkom (72 specimens, 24.82\%) followed by Ghazichak (57 specimens, 19.66\%) in Gilan Province. Eight species were only found on the southern slopes of the Alborz Mountains with low populations (Table 2). Our findings showed that $M$. tuberculifer with $36.3 \%$ and $M$. spectabilis with $36.9 \%$ of all collected specimens were the most commonly collected species. Microplitis tuberculifer was widely distributed in most locations and seems to be a common species in northern Iran. Of the five provinces sampled in this research, Guilan yielded more than $50 \%$ of the total number of collected specimens (Table 2). The 24 species of Microplitis recorded from Iran represent $22.2 \%$ of all recorded species in the Palaearctic region (108 species). The number of species of Microplitis in countries adjacent to Iran is recorded as: 58 species in Russia, 30 species in Turkey, 17 species in Turkmenistan, three species in Afghanistan and four species in Tajikistan (Fernandez-Triana et al. 2020).

The genus Microplitis is a difficult group for taxonomic studies because its species are similar and in most of the available keys related to this genus in the Palaearctic region, the species are usually separated by colouration of the body (e.g., Papp 1984; Tobias 1986). Adding DNA barcoding data will improve the accuracy of identifications in the future.

Since Iran is a large country with various geographical regions, additional new records and species are expected to be found from the region in future studies, and thus this checklist will need to be periodically updated. Finally, more research on Microplitis is required to identify biological attributes for their potential use in pest control, or to protect and conserve them.

\section{Acknowledgements}

Thanks are due to the Department of Entomology, Tarbiat Modares University, for providing financial support for this study and also to the Canadian Natural Collection of Insect (CNC) in Agriculture and Agri-food Canada for providing facilities to us. We are thankful to Drs M. Khayrandish, A. Nadimi and A. Mohammadi for helping with the collection of the specimens. We cordially thank section editor, Dr Gavin Broad (Natural History Museum, London), and an anonymous reviewer for their valuable comments and recommendations on the earlier version of this paper. Many thanks also to Dr C. van Achterberg for his valuable guidance. The senior author especially thanks Caroline Boudreault (CNC) for her help with this project.

\section{References}

Abdinbekova A.A. 1969. Braconids (Hymenoptera, Braconidae) of the lesser Caucasus in Azerbaidzan. Izvestiya Akademii Nauk Azerbaidzhanskoi SSR 5: 69-76. [In Russian.]

Abdoli P., Talebi A.A. \& Farahani S. 2019a. Dolichogenidea fernandeztrianai sp. nov. (Hymenoptera: Braconidae, Microgastrinae) from Iran. Journal of Agricultural Science and Technology 21 (3): 647658.

Abdoli P., Talebi A.A., Farahani S. \& Fernandez-Triana J. 2019b. Three new species of the genus Choeras Mason, 1981 (Hymenoptera: Braconidae, Microgastrinae) from Iran. Zootaxa 4545 (1): 77-92. https://doi.org/10.11646/zootaxa.4545.1.4

Ameri A., Talebi A.A., Beyarslan A., Kamali K. \& Rakhshani E. 2014. Study of the genus Bracon Fabricius, 1804 (Hymenoptera: Braconidae) of southern Iran with description of a new species. Zootaxa 3754 (4): 353-380. https://doi.org/10.11646/zootaxa.3754.4.2 
Ameri A., Talebi A.A., Rakhshani E., Beyarslan A. \& Kamali K. 2015. Additional evidence and new records of the genus Bracon Fabricius, 1804 (Hymenoptera: Braconidae) in southern Iran. Turkish Journal of Zoology 39: 1110-1120. https://doi.org/10.3906/zoo-1404-62

Ashmead W.H. 1906. Descriptions of new Hymenoptera from Japan. Proceedings of the United States National Museum 30: 169-201. https://doi.org/10.5479/si.00963801.30-1448.169

Austin A.D. \& Dangerfield P.C. 1993. Systematics of Australian and New Guinean Microplitis Foerster and Snellenius Westwood (Hymenoptera: Braconidae: Microgastrinae), with a review of their biology and host relationships. Invertebrate Taxonomy 7 (5): 1097-1166. https://doi.org/10.1071/IT9931097

Belokobylskij S.A., Samartsev K.G. \& Il'inskaya A.S. 2019. Annotated catalogue of the Hymenoptera of Russia. Volume II. Apocrita: Parasitica. Proceedings of the Zoological Institute RAS 323 (Suppl. 8): 1-594. https://doi.org/10.31610/trudyzin/2019.supl.8.5

Bouche P.F. 1834. Naturgeschichte der Insekten, besonders in Hinsicht ihrer ersten Zustande als Larven und Puppen. Nicolaische Buchhandlung, Berlin.

Brewster J.D. \& Paoli G.C. 2013. DNA extraction protocol for rapid PCR detection of pathogenic bacteria. Analytical Biochemistry 442: 107-109. https://doi.org/10.1016/j.ab.2013.07.013

Broad G.R., Shaw M.R. \& Godfray H.C.J. 2016. Checklist of British and Irish Hymenoptera-Braconidae. Biodiversity Data Journal 4: e8151. https://doi.org/10.3897/BDJ.4.e8151

Cameron P. 1906. On the Tenthredinidae and parasitic Hymenoptera collected in Baluchistan by Major C.G. Nurse. Part I. Journal of the Bombay Natural History Society 17: 89-107.

Chen J. \& Song D. 2004. Systematic Studies on Microgastrinae of China (Hymenoptera: Braconidae). Fujian Scientific Publisher, Fuchow.

De Saeger H. 1944. Microgastrinae (Hymenoptera: Apocrita). Exploration du Parc national Albert. Mission G.F. de Witte 47:1-342.

Fabricius J.C. 1798. Supplementum entomologiae systematicae. Proft \& Storch, Hafnia [Copenhagen].

Fahringer J. 1937. Opuscula braconologica. Band 4. Palaearktische Region. Lieferung 4-6. In: Opuscula braconologica: 257-520. Verlag Von Fritz Wagner, Wien [Vienna].

Farahani S., Talebi A.A., Rakhshani E. \& van Achterberg C. 2012. First record of Homolobus infumator (Lyle, 1914) (Insecta: Hymenoptera: Braconidae: Homolobinae) from Iran. Check List 8: 1350-1352. https://doi.org/10.15560/8.6.1350

Farahani S., Talebi A.A. \& van Achterberg C. 2014a. A review of species of the genus Ascogaster Wesmael (Hymenoptera: Braconidae, Cheloninae) from Iran. Far Eastern Entomologist 275: 1-12.

Farahani S., Talebi A.A., van Achterberg C. \& Rakhshani E. 2014b. A new species of the genus Mirax Haliday, 1833 (Hymenoptera: Braconidae: Miracinae) from Iran. Annales Zoologici 64: 677682. https://doi.org/10.3161/000345414X685956

Farahani S., Talebi A.A., van Achterberg C. \& Rakhshani E. 2014c. First record of Microplitis rufiventris Kokujev, 1914 (Braconidae: Microgastrinae) from Iran. Check List 10: 441-444.

https://doi.org/10.15560/10.2.441

Farahani S., Talebi A.A. \& Rakhshani E. 2014d. Wasps of the subfamily Doryctinae (Hymenoptera: Braconidae) in Iran. Zoology in the Middle East 60: 65-81.

https://doi.org/10.1080/09397140.2014.892341

Farahani S., Talebi A.A., van Achterberg C. \& Rakhshani E. 2015. A review of the subfamily Rogadinae (Hymenoptera: Braconidae) from Iran. Zootaxa 3973: 227-250.

https://doi.org/10.11646/zootaxa.3973.2.2 
Farahani S., Talebi A.A \& Rakhshani E. 2016. Iranian Braconidae (Insecta: Hymenoptera: Ichneumonoidea): diversity, distribution and host association. Journal of Insect Biodiversity and Systematics 2: 1-92.

Fernandez-Triana J., Whitfield J.B., Smith M.A., Kula R., Hallwachs W. \& Janzen D. 2015. Revision of the genera Microplitis and Snellenius (Hymenoptera, Braconidae, Microgastrinae) from Area de Conservacion Guanacaste, Costa Rica, with a key to all species previously described from Mesoamerica. Deutsche entomologische Zeitschrift 62 (2): 137-201. https://doi.org/10.3897/dez.62.5276.

Fernandez-Triana J., Shaw M.R., Boudreault C., Beaudin M. \& Broad G.R. 2020. Annotated and illustrated world checklist of Microgastrinae parasitoid wasps (Hymenoptera, Braconidae). ZooKeys 920: 1-1089. https://doi.org/10.3897/zookeys.920.39128

Flomer O., Black M., Hoeh W., Lutz R. \& Vrijenhoek R. 1994. DNA primers for amplification of mitochondrial cytochrome c oxidase subunit I from diverse metazoan invertebrates. Molecular Marine Biology and Biotechnology 3: 294-299.

Gadallah N.S., Ghahari H. \& Peris-Felipo F.J. 2015. Catalogue of the Iranian Microgastrinae (Hymenoptera: Braconidae). Zootaxa 4043: 1-69. https://doi.org/10.11646/zootaxa.4043.1.1.

Gautier C. \& Bonnamour S. 1939. Hyménoptères parasites nouveaux ou peu connus. Bulletin Mensuel de la Societe Linneenne de Lyon 8: 190-191. https://doi.org/10.3406/linly.1939.9492

Ghafouri Moghaddam M., Rakhshani E., van Achterberg C. \& Mokhtari A. 2018. A study of the Iranian species of Choeras Mason (Hymenoptera: Braconidae: Microgastrinae), with the description of a new species. Zootaxa 4446 (4): 455-476. https://doi.org/10.11646/zootaxa.4446.4.3.

Ghahari H. \& Beyarslan A. 2017. A faunistic study on Braconidae (Hymenoptera: Ichneumonoidea) from Iran. Natura Somogyiensis 30: 39-46.

Ghahari H. Fischer M. \& Papp J. 2011a. A study on the Braconidae (Hymenoptera: Ichneumonoidea) from Ilam province, Iran. Calodema 160: 1-5.

Ghahari H., Fischer M. \& Papp J. 2011b. A study on the Braconidae (Hymenoptera: Ichneumonoidea) from Qazvin province, Iran. Entomofauna 32: 197-204.

Ghahari H., Fischer M. \& Papp J. 2011c. A study on the braconid wasps (Hymenoptera: Braconidae) from Isfahan province, Iran. Entomofauna 32: 261-272.

Ghahari H., Fischer M., Sakenin H. \& Imani S. 2011d. A contribution to the Agathidinae, Alysinae, Aphidiinae, Braconinae, Microgastrinae and Opiinae (Hymenoptera: Braconidae) from cotton fields and surrounding grasslands of Iran. Linzer biologische Beiträge 43: 1269-1276.

Haliday A.H. 1834. Essay on parasitic Hymenoptera. Entomological Magazine 2 (3): 225-259.

Ivanov P. 1899. Braconids cryptogastres et aréolaires des environs de Koupiansk avec tableaux synoptiques des genres et des espèces de ces insectes. Trudy Obshchestva Ispytatelei Prirody pri Imperatorskom Khar 'kovskom Universitete 33: 273-382. [In Ukrainian.]

Karimi-Malati A., Fathipour Y., Talebi A.A. \& Lozan A.I. 2014. The first report of Microplitis fulvicornis (Hym.: Braconidae: Microgastrinae) as a parasitoid of Spodoptera exigua (Lep.: Noctuidae) from Iran. Journal of Entomological Society of Iran 33: 71-72.

Karlsson D. \& Ronquist F. 2012. Skeletal morphology of Opius dissitus and Biosteres carbonarius (Hymenoptera: Braconidae), with a discussion of terminology. PLoS One 7 (4): e32573.

https://doi.org/10.1371/journal.pone.0032573 
Kiani M., Mohammadi S., Babaei A., Sefidkon F., Naghavi M.R., Ranjbar M., Razavi S.A., Saeidi K., Jafari H., Asgari D. \& Potter D. 2017. Iran supports a great share of biodiversity and floristic endemism for Fritillaria spp. (Liliaceae): A review. Plant Diversity 39: 245-262.

https://doi.org/10.1016/j.pld.2017.09.002

Kokujev N.R. 1914. Hymenoptera parasitic nove fauna turanica V.I. Platnikov collecta. Revue Russe d'Entomologie 13: 513-514.

Kotenko A.G. 2007. Microgastrinae. In: Lelej A.S. (ed.) Key to the Insects of Russia Far East. Vol. IV. Neuropteroidea, Mecoptera, Hymenoptera. Pt 5: 134-192. Dalnauka, Vladivostok. [In Russian.]

Marshall T.A. 1898. Description de Braconides. Bulletin du Museum national d'Histoire naturelle, Paris 4: 369-371

Marshall T.A. 1900. Les Braconides (Supplement). In: Andre E. (ed.) 1897-1900. Species des Hyménoptères d'Europe et d'Algérie. Tome 5 bis. Paris.

Mason W.R.M. 1981. The polyphyletic nature of Apanteles Foerster (Hymenoptera: Braconidae): A phylogeny and reclassification of Microgastrinae. Memoirs of the Entomological Society of Canada 113: 1-147. https://doi.org/10.4039/entm113115fv

Nees von Esenbeck C.G. 1834. Hymenopterorum Ichneumonibus affinium monographiae, genera Europaea et species illustrantes. Vol. 1. J.G. Cottae, Stuttgart/Tübingen.

https://doi.org/10.5962/bhl.title.26555

Nixon G.E.J. 1968. A revision of the genus Microgaster Latreille (Hymenoptera: Braconidae). Bulletin of the British Museum (Natural History), Entomology series 22: 33-72.

https://doi.org/10.5962/bhl.part.9950

Nixon G.E.J. 1970. A revision of the N.W. European species of Microplitis Förster (Hymenoptera: Braconidae). Bulletin of the British Museum (Natural History), Entomology series 25: 1-30.

Papp J. 1980. Braconidae (Hymenoptera) from Mongolia VIII. Acta Zoologica Hungarica 26:401-413.

Papp J. 1984. Palaearctic species of Microgaster Latreille (= Microplitis Förster) with description of seven new species (Hymenoptera, Braconidae, Microgastrinae). Entomologische Abhandlungen 47: $95-140$.

Papp J. 1986. First survey of the Glabromicroplitis Papp species of the Holarctic region, with taxonomical remarks of three Microgaster Latreille species (Hymenoptera, Braconidae: Microgastrinae). Annales Historico-Naturales Musei Nationalis Hungarici 78: 249-253.

Papp J. 1987. First survey of the braconid fauna of the Kiskunsäg National Park, Hungary (Hymenoptera, Braconidae). The Fauna of the Kiskunsäg National Park 2: 314-334.

Papp J. 1990. Second survey of the braconid wasps in the Bátorliget Nature Conservation Areas, Hungary (Hymenoptera: Braconidae). Studia Naturalia. In: Mahunka S. (ed.) The Bátorliget Nature Reserves after Forty Years: 639-674. Magyar Természettudományi Múzeum, Budapest.

Prell H. 1925. Zur Biologie eines bisher verkannten Kieferneulenschmarotzers (Microplitis decipiens sp.n.). Zeitschrift für wissenschaftliche Insektenbiologie 20: 137-147.

Rakhshani E., Talebi A.A., Stary P.S., Tomanović Z.T. \& Manzari S.M. 2007. Aphid-Parasitoid (Hymenoptera, Braconidae, Aphidiinae) Associations on Willows and Poplars in Iran. Acta Zoologica Academiae Scientiarum Hungaricae 53: 281-292.

Ranjith A.P., Rajesh K.M. \& Nasser M. 2015. Taxonomic studies on Oriental Microplitis Foerster (Hymenoptera: Braconidae, Microgastrinae) with description of two new species from south India. Zootaxa 3963: 369-415. https://doi.org/10.11646/zootaxa.3963.3.4 
Rastegar J., Sakenin H., Khodaparast S. \& Havaskary M. 2012. On a collection of Braconidae (Hymenoptera) from East Azarbaijan and vicinity, Iran. Calodema 226: 1-4.

Reinhard H. 1880. Beiträge zur Kenntniss einiger Braconiden-Gattungen. Fünftes Stück. XVI. Zur Gattung Microgaster Latr. (Microgaster, Microplitis, Apanteles). Deutsche entomologische Zeitschrift 24: $353-370$.

Rodriguez J.J., Fernandez-Triana J.L., Smith M.A., Janzen D.H., Hallwachs W., Erwin T.L. \& Whitfield J.B. 2013. Extrapolations from field studies and known faunas converge on dramatically increased estimates of global Microgastrine parasitoid wasp species richness (Hymenoptera: Braconidae). Insect Conservation and Diversity 6: 530-536. https://doi.org/10.1111/icad.12003

Ruthe J.F. 1860. Deutsche Braconiden. Erstes Stück. Berliner entomologische Zeitschrift 4: 105-160.

Samin N., Coronado-Blanco J.M., Fischer M., van Achterberg C., Sakenin H. \& Davidian E. 2018. Updated checklist of Iranian Braconidae (Hymenoptera: Ichneumonoidea) with twenty-three new records. Natura Somogyiensis 30 (32): 21-36.

Samin N., Coronado-Blanco J.M., Hosseini A., Fischer M. \& Sakenin Chelav H. 2019. A faunistic study on the braconid wasps (Hymenoptera: Braconidae) of Iran. Natura Somogyiensis 33: 78-80. https://doi.org/10.24394/NatSom.2019.33.75

Schulz W.A. 1906. Spolia Hymenopterologica. Junfermann, Paderborn. https://doi.org/10.5962/bhl.title.59757

Shaw M.R. \& Huddleston T.B. 1991. Classification and biology of Braconid Wasps. Royal Entomological Society of London 7 (11): 1-126.

Shorthouse D.P. 2010. SimpleMappr, an online tool to produce publication-quality point maps. Available from https://www.simplemappr.net [accessed 23 Feb. 2020].

Smith M.A., Fernández-Triana J.L., Eveleigh E., Gomez J., Guclu C., Hallwachs W., Hebert P.D., Hrcek J., Huber J.T., Janzen D., Mason P.G., Miller S., Quicke D.L.J., Rodriguez J.J., Rougerie R., Shaw M.R., Várkonyi G., Ward D.F., Whitfield J.B. \& Zaldívar-Riverón A. 2013. DNA barcoding and the taxonomy of Microgastrinae wasps (Hymenoptera, Braconidae): impacts after 8 years and nearly 20000 sequences. Molecular Ecology Resources 13 (2): 168-176. https://doi.org/10.1111/1755-0998.12038

Szépligeti G. 1896. Beiträge zur Kenntnis der Ungarischen Braconiden. (Zweiter Theil). Természetrajzi Füzetek 19: 285-321 [In Hungarian.], 359-386 [In German.].

Szépligeti G. 1898. Beiträge zur Kenntnis der Ungarischen Braconiden, 3. Theil. Természetrajzi Füzetek 21: 381-396 [In Hungarian.], 396-408 [In German.].

Telenga N.A. 1955. Braconidae, subfamily Microgastrinae, subfamily Agathinae. Fauna USSR, Hymenoptera. Zoological Institute of the Academy of Science of the U.S.S.R. 5 (4): 1-311.

Thomson C.G. 1895. LII. Bidrag till Braconidernas Kännedom. Opuscula Entomologica 20: 2141-2339.

Tobias V.I. 1964. New species and genus of Braconids (Hymenoptera, Braconidae) from Kazakhstan. Trudy Zoologicheskogo Instituta. Leningrad 34: 177-234.

Tobias V.I. 1976. Microgastrinae (Hymenoptera: Braconidae) of Caucasus: 156-258. SSSR Science Academy Zoology Institute ELM.

Tobias V.I. 1986. Subfamily Microgastrinae. In: Medvedev G.S. (ed.) Keys to the Insects of the European Part of the USSR. Volume III. Part IV. Amerind Publishing Co., New Delhi.

Wesmael C. 1837. Monographie des Braconides de Belgique. (Suite.) Nouveaux Mémoires de l'Academie royale des Sciences et Belles-Lettres de Bruxelles 10: 1-68. 
Wharton R.A., Marsh P.M. \& Sharkey M.J. 1997. Manual of the New World Genera of the Family Braconidae (Hymenoptera). Special Publication of the International Society of Hymenopterists, Washington DC.

Whitfield J.B., Mardulyn P., Austin A.D. \& Dowton M. 2002. Phylogenetic relationships among Microgastrine braconid wasp genera based on data from the 16S, COI and 28S genes and morphology. Systematic Entomology 27: 337-359. https://doi.org/10.1046/j.1365-3113.2002.00183.x

Xu W.-A. \& He J.-H. 2003. Two new species of Microplitis Foerster from China (Hymenoptera, Braconidae, Microgastrinae). Acta Zootaxonomica Sinica 28: 724-728.

Zargar M., Gupta A., Talebi A.A. \& Farahani S. 2019a. Description of a new species of the genus Deuterixys (Hymenoptera: Braconidae: Microgastrinae) from Iran. Biologia 1-6.

https://doi.org/10.2478/s11756-019-00298-9

Zargar M., Gupta A., Talebi A.A. \& Farahani S. 2019b. A review of the Iranian species of genus Iconella Mason (Hymenoptera: Braconidae: Microgastrinae) with description of two new species. Zootaxa 4586 (3): 491-504. https://doi.org/10.11646/zootaxa.4586.3.6

Zargar M., Gupta A., Talebi A.A. \& Farahani S. 2019c. Three new species and two new records of the genus Cotesia Cameron (Hymenoptera: Braconidae) from Iran. European Journal of Taxonomy 571: 1-25. https://doi.org/10.5852/ejt.2019.571

Zhang W., Song D. \& Chen J. 2019. Two new species of the genus Microplitis Förster, 1862 (Hymenoptera, Braconidae, Microgastrinae) from China. ZooKeys 859: 49-61.

https://doi.org/10.3897/zookeys.859.31720

Manuscript received: 25 July 2020

Manuscript accepted: 20 December 2020

Published on: 9 April 2021

Topic editor: Nesrine Akkari

Section editor: Gavin Broad

Desk editor: Radka Rosenbaumová

Printed versions of all papers are also deposited in the libraries of the institutes that are members of the EJT consortium: Muséum national d'histoire naturelle, Paris, France; Meise Botanic Garden, Belgium; Royal Museum for Central Africa, Tervuren, Belgium; Royal Belgian Institute of Natural Sciences, Brussels, Belgium; Natural History Museum of Denmark, Copenhagen, Denmark; Naturalis Biodiversity Center, Leiden, the Netherlands; Museo Nacional de Ciencias Naturales-CSIC, Madrid, Spain; Real Jardín Botánico de Madrid CSIC, Spain; Zoological Research Museum Alexander Koenig, Bonn, Germany; National Museum, Prague, Czech Republic. 